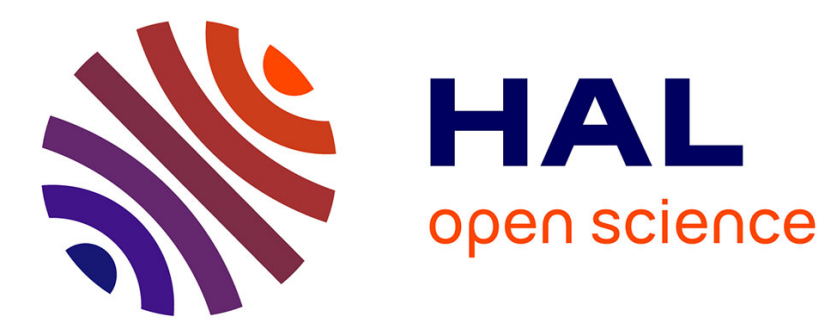

\title{
Extended Magneto-Hydro-Dynamic model for Neoclassical Tearing Mode Computations
}

Patrick Maget, Olivier Février, Xavier Garbet, Hinrich Lütjens, Jean-Francois Luciani, Alain Marx

\section{- To cite this version:}

Patrick Maget, Olivier Février, Xavier Garbet, Hinrich Lütjens, Jean-Francois Luciani, et al.. Extended Magneto-Hydro-Dynamic model for Neoclassical Tearing Mode Computations. Nuclear Fusion, 2016, 56 (8), pp.086004. 10.1088/0029-5515/56/8/086004 . hal-01342244

\section{HAL Id: hal-01342244 \\ https://hal.science/hal-01342244}

Submitted on 5 Jul 2016

HAL is a multi-disciplinary open access archive for the deposit and dissemination of scientific research documents, whether they are published or not. The documents may come from teaching and research institutions in France or abroad, or from public or private research centers.
L'archive ouverte pluridisciplinaire HAL, est destinée au dépôt et à la diffusion de documents scientifiques de niveau recherche, publiés ou non, émanant des établissements d'enseignement et de recherche français ou étrangers, des laboratoires publics ou privés. 


\title{
Extended Magneto-Hydro-Dynamic model for Neoclassical Tearing Mode Computations
}

\author{
Patrick Maget, Olivier Février, Xavier Garbet, Hinrich Lütjens ${ }^{1}$, Jean-Francois Luciani ${ }^{1}$, Alain Marx ${ }^{1}$ \\ CEA, IRFM, F-13108 Saint Paul-lez-Durance, France. \\ ${ }^{1}$ Centre de Physique Théorique, Ecole Polytechnique, CNRS, France.
}

patrick.maget@cea.fr

\begin{abstract}
A self-consistent fluid model for describing Neoclassical Tearing Modes in global Magneto-HydroDynamic simulations is presented. It is illustrated by its application to a simple toroidal configuration unstable to the $(2,1)$ tearing mode. The island saturation is verified to increase with the bootstrap current fraction. New features that are specific to this model are evidenced, like the unsteady saturated state of the island, and its deformation to a droplet shape, when the magnetic Prandtl number is not too high. Synthetic diagnostics demonstrate that diamagnetic and neoclassical effects should have in this case a measurable impact on the signature of magnetic islands.
\end{abstract}

\section{Introduction}

Magnetic islands can grow in the plasma of magnetic confinement devices and cause a degradation of the energy confinement time Sauter et al. (1997) that could impact the fusion gain of a fusion reactor Halpern et al. (2006). As pressure is increased, their linear stability is improved Glasser et al. (1975); Lütjens et al. (2001), but a metastable branch emerges due to the nonlinear drive provided by neoclassical friction forces Carrera et al. (1986). Islands produced by this mechanism are called Neoclassical Tearing Modes Chang et al. (1995), and their suppression by real-time control systems is crucial for present and future high performance tokamaks Maraschek (2012).

In view of predicting the threshold and saturation of Neoclassical Tearing Modes (NTM), several approaches have been adopted. The simplest one is to use the simplified formalism of the Rutherford Equation Rutherford (1973), where the contributions of various physical effects on the evolution of the island width can be implemented provided the asymptotic matching procedure is valid (i.e. the island width remains small compared to equilibrium plasma scales). Neoclassical physics can be inserted in a Generalized Rutherford Equation using either fluid Smolyakov et al. (1995) or kinetic approaches Wilson et al. (1996).

Another solution is to compute NTM dynamics from a global code where the plasma fields are evolved without scale separation. This allows removing the small island limitation of the Rutherford equation framework as well as the uncertainties related to the modelling of the island saturation Arcis et al. (2007); Maget et al. (2010), and integrating self-consistently toroidal effects. The drive for NTMs being due to the perturbation of the self-generated bootstrap current by the island, an ad-hoc implementation of this current into the single fluid (1F) resistive MHD model has been first used Yu and Günter (1998); Popov et al. (2002); Lütjens and Luciani (2002); Maget et al. (2010). However, the importance of diamagnetic rotations for the dynamics of magnetic islands Ara et al. (1978); Yu (2010) claims for the use of a bifluid MHD model covering self-consistently neoclassical physics. The formal derivation of such a model, proposed in Callen et al. (1986); Callen (2010), has been implemented in the XTOR code Lütjens and Luciani (2010). Equilibrium flows and bootstrap current are formally recovered, and the nonlinear NTM threshold can be computed with this model, as shown in Mellet et al. (2013). In that first application, parallel heat flows were not considered, and their proposed formulation was incomplete. We detail in the present work how these flows can be implemented, and we show that their contribution to the bootstrap current is essential.

The paper first describes the Magneto-Hydro-Dynamics (MHD) model that is used in the computations (section 2). The application to a circular cross-section plasma with an unstable tearing mode is then presented, and the main characteristics of the saturated state are described (section 3). 


\section{Magneto-Hydro-Dynamics model for Neoclassical Tearing Mode simulations}

\subsection{The fluid equations}

The two-fluid neoclassical model implemented in XTOR solves the following normalized equations Lütjens and Luciani (2010):

$$
\begin{aligned}
\left(\partial_{t}+\mathbf{V}_{\mathbf{i}} \cdot \nabla\right) \rho= & -\rho \nabla \cdot \mathbf{V}_{\mathbf{i}}-\nabla \cdot \Gamma_{a n}+\Sigma \\
\left(\partial_{t}+\mathbf{V} \cdot \nabla\right) p= & -\Gamma p \nabla \cdot \mathbf{V}-d_{i} \Gamma \mathbf{K} \cdot\left[\frac{p}{\rho} \nabla p_{i}+\frac{p_{i}}{\rho} \nabla p_{i}-\frac{p_{e}}{\rho} \nabla p_{e}+\frac{p_{e}^{2}-p_{i}^{2}}{\rho^{2}} \nabla \rho\right]+ \\
& +H-(\Gamma-1) \nabla \cdot \mathbf{q}_{\chi} \\
\rho\left(\partial_{t}+\mathbf{V} \cdot \nabla\right) \mathbf{V}= & -\rho \mathbf{V}_{i}^{*} \cdot \nabla \mathbf{V}_{\perp}+\mathbf{J} \times \mathbf{B}-\nabla p-\nabla \cdot \Pi_{\|}+\nabla \cdot \nu \nabla \mathbf{V}_{\mathbf{i}} \\
\partial_{t} \mathbf{B}= & -\nabla \times \mathbf{E}
\end{aligned}
$$

with $\rho$ the mass density, $p$ the total pressure, $\mathbf{K}=\nabla \times\left(\mathbf{B} / B^{2}\right), \mathbf{V}_{\mathbf{i}}=\mathbf{V}+\mathbf{V}_{i}^{*}, \mathbf{V}=\mathbf{V}_{\mathbf{E}}+\mathbf{V}_{\| \mathbf{i}}$, $\mathbf{V}_{\mathbf{E}}=\mathbf{E} \times \mathbf{B} / B^{2}$ and $\mathbf{V}_{i}^{*}=d_{i} \mathbf{B} \times \nabla p_{i} /\left(\rho B^{2}\right)$. Here, the ion skin depth $d_{i}=V_{A} /\left(a \omega_{c i}\right)\left(V_{A}=B / \sqrt{\mu_{0} n_{i} m_{i}}\right.$ and $\left.\omega_{c i}=e_{i} B / m_{i}\right)$ is a measure of diamagnetic effect. The ratio of specific heat is $\Gamma=5 / 3, H \equiv$ $-(\Gamma-1) \nabla \cdot \chi_{\perp} \nabla_{\perp} p(t=0)$ is the heat source and $\mathbf{q}_{\chi}=-\rho \chi_{\|} \mathbf{b}(\mathbf{b} \cdot \nabla T)-\rho \chi_{\perp} \nabla_{\perp} T$ is the diffusive heat flux $(\mathbf{b} \equiv \mathbf{B} / B)$, with $T=p / \rho$. This diffusive heat flux is aimed at representing the effect of turbulent processes in the perpendicular direction (leading to anomalous transport), and of collisions in the parallel direction. Ion and electron temperatures are assumed to be proportional : $T_{i}=\tau T_{e}$ with $\tau$ a constant, and we then have $p_{e}=Z_{i} \rho T_{e}$ (with $Z_{i}$ the ion charge), $p_{i}=\rho T_{i}$ and $p \equiv p_{e}+p_{i}$. The anomalous particle flux is defined as $\Gamma_{a n}=-D_{\perp} \nabla \rho+\rho \mathbf{V}_{\text {pinch }}$ with $D_{\perp}$ the perpendicular diffusion coefficient and $\mathbf{V}_{\text {pinch }}$ the pinch velocity. The introduction of a pinch velocity is aimed at suppressing the particle source in the plasma core, thus removing any artificial feeding when particles are transported by MHD activity. This pinch is vanishing in the edge region, outside a prescribed radius $y=y_{\text {ped }}$ (here $y \equiv \sqrt{\psi}$ and we prescribe $\left.y_{\text {ped }}=0.9\right)$ :

$$
\begin{aligned}
\mathbf{V}_{\text {pinch }} & =D_{\perp} \frac{\nabla \rho}{\rho} f(y) \\
f\left(y<y_{\text {ped }}\right) & =1 \\
f\left(y>y_{\text {ped }}\right) & =0.5\left(1+\cos \left(\pi \frac{y-y_{\text {ped }}}{1-y_{\text {ped }}}\right)\right)
\end{aligned}
$$

The particle source is then defined as $\Sigma=\nabla \cdot\left(-D_{\perp} \nabla \rho+\rho \mathbf{V}_{\text {pinch }}\right)$ and it vanishes inside $y_{\text {ped }}$, as required. The pinch velocity, as well as the density source, are defined from the initial equilibrium, and are not evolved.

The system of equation is completed by the Ohm's law:

$$
\mathbf{E}+\mathbf{V} \times \mathbf{B}=\eta\left[\mathbf{J}-\mathbf{J}_{\mathbf{b s}}-\mathbf{J}_{\mathbf{C D}}\right]-d_{i} \frac{\nabla_{\|} p_{e}}{\rho}
$$

with $\mathbf{J}_{\mathbf{C D}}$ the current density source, and $\mathbf{J}_{\mathbf{b s}} \equiv J_{b s} \mathbf{b}$ the bootstrap current. The bootstrap current, whose expression is given in the forthcoming equation (19), can be amplified by a prescribed factor $f_{b s}$ in the simulations. We detail in the following sections how $J_{b s}$ and the neoclassical force $\nabla \cdot \Pi_{\|}$are precisely implemented. Note that polarization physics is only partly taken into account because the polarization drift is not yet included in the density evolution (for this, a density evolution equation based on the electron population should be implemented). The MHD model that we obtain covers drift-tearing and neoclassical physics in a consistent way.

\subsection{Implementation of neoclassical physics}

The neoclassical model implemented in XTOR has been first described in Mellet et al. (2013), and applied in different regimes in Maget et al. $(2013,2014)$. We have recently significantly improved the 
implementation of parallel heat fluxes, and the numerical robustness of the model, as explained in the following. In particular, the formulation proposed here differs from the initial one proposed in Mellet et al. (2013) regarding the contribution of parallel heat fluxes, which was incorrectly formulated (but not used in simulations so far). Indeed, they cannot be used as flux-averaged as proposed in that paper, because the contribution of perturbed quantities would then not be self-consistent.

The stress tensor is expressed in a CGL form as

$$
\begin{aligned}
\Pi_{\|} & =\frac{3}{2} \pi_{\|}\left[\mathbf{b} \mathbf{b}-\frac{1}{3} \mathbf{I}\right] \\
\frac{3}{2} \pi_{\|} & =p_{\|}-p_{\perp}
\end{aligned}
$$

so that

$$
\nabla \cdot \Pi_{\|}=\frac{3}{2} \pi_{\|}[(\nabla \cdot \mathbf{b}) \mathbf{b}+\boldsymbol{\kappa}]+\left(\mathbf{b} \cdot \nabla \frac{3}{2} \pi_{\|}\right) \mathbf{b}-\frac{1}{3} \nabla \frac{3}{2} \pi_{\|}
$$

with $\nabla \cdot \mathbf{b}=-\nabla_{\|} \ln B$ and $\boldsymbol{\kappa} \equiv \mathbf{b} \cdot \nabla \mathbf{b}$ the magnetic curvature.

The perpendicular heat flow is related to the temperature drift velocity by $2 \mathbf{q}_{\perp \mathbf{s}} /\left(5 p_{s}\right)=\mathbf{V}_{\mathbf{T s}}^{*}$, with $\mathbf{V}_{T s}^{*}=d_{i} / Z_{s} \mathbf{B} \times \nabla T_{s} / B^{2}$, and for the parallel heat flow, we use the notation

$$
u_{2 \|, s}=\frac{2}{5 p_{s}} \mathbf{q}_{\mathbf{s}} \cdot \mathbf{B}
$$

Note that, in contrast with the diffusive heat flux previously introduced $\left(\mathbf{q}_{\chi}\right)$ that represents turbulent and collisional processes, the heat flow $\mathbf{q}_{\mathbf{s}}$ derives from the third moment of the Vlasov equation Hirshman and Sigmar (1981), and is of convective origin.

The electron dynamics is described in the Ohm's law, where electron inertia is neglected:

$$
\mathbf{E}+\mathbf{V} \times \mathbf{B}=\left(d_{i} / \rho\right) \mathbf{R}_{\mathbf{e} \|}-\left(d_{i} / \rho\right) \mathbf{b}\left[\mathbf{b} \cdot \nabla \cdot\left(p_{e} \mathbf{I}+\Pi_{\| e}\right)\right]
$$

with $\mathbf{R}_{\mathbf{e} \|}$ the parallel friction force exerted on electrons:

$$
\left(d_{i} / \rho\right) \mathbf{R}_{\mathbf{e} \|}=\alpha_{e} d_{i} \sum_{s}\left[\Lambda_{11}^{e s} \mathbf{V}_{\mathbf{s} \|}+\Lambda_{12}^{e s} \frac{u_{2 \|, s}}{B^{2}} \mathbf{B}\right]
$$

with $\alpha_{s}=\left(Z_{i} m_{s}\right) /\left(Z_{s} m_{i}\right), Z_{s}$ the charge number of the species 's', and $\Lambda_{i j}^{s s^{\prime}}$ are the friction coefficients for Coulomb collisions (see Hirshman and Sigmar (1981), with the correspondence $l_{i j}^{s s^{\prime}}=n_{s} m_{s} \Lambda_{i j}^{s s^{\prime}}$ in real units). Parallel heat fluxes are computed in the assumption that they are at equilibrium at any time. They can therefore be expressed as linear combinations of parallel velocities and thermodynamical sources:

$$
\frac{u_{2 \|, s}}{B}=\sum_{s^{\prime}}\left[C_{\|}^{s s^{\prime}} V_{s^{\prime} \|}+C_{1}^{s s^{\prime}} S_{1 \theta}^{s^{\prime}}+C_{2}^{s s^{\prime}} S_{2 \theta}^{s^{\prime}}\right]
$$

where

$$
\begin{aligned}
& S_{1 \theta}^{s^{\prime}}=C(\mathbf{B} \cdot \nabla \ln B)\left(\mathbf{V}_{\mathbf{s}}^{*}+\mathbf{V}_{\mathbf{E}}\right) \cdot \nabla \ln B \\
& S_{2 \theta}^{s^{\prime}}=C(\mathbf{B} \cdot \nabla \ln B) \mathbf{V}_{\mathbf{T} \mathbf{s}}^{*} \cdot \nabla \ln B
\end{aligned}
$$

are a generalization of the neoclassical source terms (see appendix) and $C \equiv B^{2} /\left\langle(\mathbf{b} \cdot \nabla B)^{2}\right\rangle$. The expressions for coefficients $C_{\|}^{s s^{\prime}}, C_{1}^{s s^{\prime}}$ and $C_{2}^{s s^{\prime}}$ are given in appendix.

The scalar pressure is defined as $p=\left(p_{\|}+2 p_{\perp}\right) / 3$, so that

$$
\mathbf{b} \cdot \nabla\left(p_{e} \mathbf{I}+\Pi_{\| e}\right)=\frac{3}{2} \pi_{\| e} \nabla \cdot \mathbf{b}+\nabla_{\|} p_{\| e}
$$

The boostrap current can then be expressed as

$$
J_{b s}=\frac{\eta-\eta_{S P}}{\eta}\left[J_{\|}+\frac{d_{i}}{\rho} \frac{3 / 2 \pi_{\| e}}{\left(\eta-\eta_{S P}\right)} \nabla \cdot \mathbf{b}-\alpha_{e} \frac{d_{i}}{\eta-\eta_{S P}} \sum_{s} \Lambda_{12}^{e s} \frac{u_{2 \|, s}}{B}\right]
$$




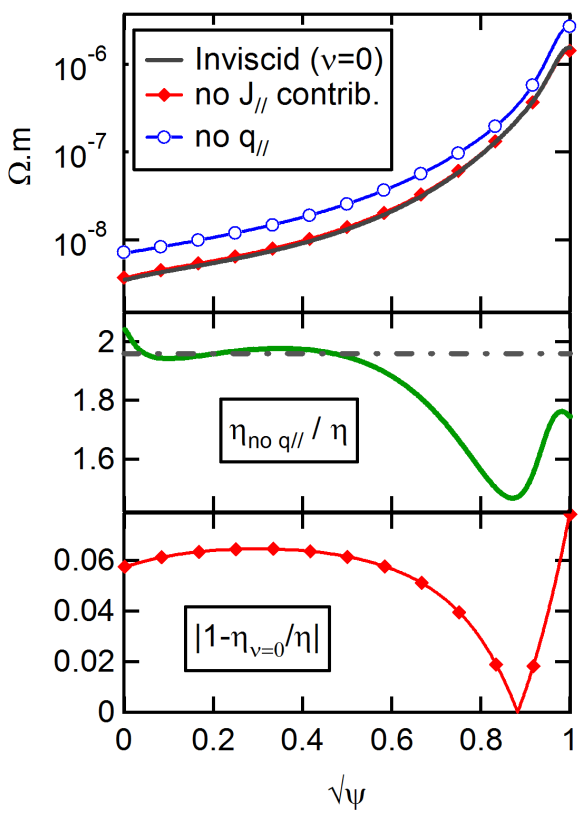

Figure 1: Top: Neoclassical resistivity from the inviscid neoclassical problem, using the constraint of null $J_{\|}$contribution (equation 20), and without the parallel heat flows contribution. Middle: Ratio between the plasma resistivity without and with parallel heat flows. Bottom: Relative difference between the neoclassical resistivity (equation 20) and the inviscid neoclassical solution $\eta_{\nu=0}$.

where the last sum is performed over the two species, ion and electron, and $\eta_{S P} \equiv \alpha_{e} d_{i}^{2} \Lambda_{11}^{e i} / \rho$ is the Spitzer resistivity. The expression for the neoclassical resistivity $\eta$ can be obtained by imposing that $J_{b s}$ does not depend explicitly on the plasma current $J_{\|}$. When parallel heat flows are taken into account, this leads to

$$
\eta \equiv \alpha_{e} \frac{d_{i}^{2}}{\rho}\left[\mu_{1}^{e}+\Lambda_{11}^{e i}+\left(\mu_{2}^{e}-\Lambda_{12}^{e e}\right) C_{\|}^{e e}-\Lambda_{12}^{e i} C_{\|}^{i e}\right]
$$

This formulation of plasma resistivity is very close to the exact solution of the neoclassical equilibrium problem as formulated in Hirshman and Sigmar (1981); Kessel (1994) or Houlberg et al. (1997). It is not exactly equal to it because in the neoclassical equilibrium problem one has an additional relation between $V_{\|}$and $J_{\|}$from the momentum equation where viscous effects have been neglected, and we do not have this explicit relation. For the example that will be described later, the difference is however less than $10 \%$ (figure 1). In the case where parallel heat flows are neglected, we need to consider

$$
\eta_{n o q_{\|}} \equiv \alpha_{e} \frac{d_{i}^{2}}{\rho}\left[\mu_{1}^{e}+\Lambda_{11}^{e i}\right]
$$

In this latter expression, neoclassical resistivity is evaluated by adding the neoclassical friction to the electron-ion collision frequency in the Spitzer estimate $\eta_{S P}$. The plasma resistivity thus obtained, where parallel heat flows have been neglected, is about a factor of two above the resistivity computation with parallel heat flows. This corresponds to the ratio between perpendicular and parallel resistivities Wesson (1997) (figure 1).

In conditions where the pressure anisotropy is small compared to the scalar pressure, we can identify $p_{\| e}$ with $p_{e}$ in equation (18). Compared to the initial implementation Mellet et al. (2013), this one has a better numerical behaviour thanks to the incorporation of $\nabla_{\|} \pi_{\| e}$ in the scalar pressure. The Ohm's law finally writes as shown in equation 8 . 
For the momentum equation, we obtain similarly

$$
\nabla p+\nabla \cdot\left(\Pi_{i}+\Pi_{e}\right)=\left(p_{\|}-p_{\perp}\right)[(\nabla \cdot \mathbf{b}) \mathbf{b}+\boldsymbol{\kappa}]+\nabla_{\|}\left(p_{\|}-p_{\perp}\right)+\nabla p_{\perp}
$$

and for situations where the pressure anisotropy is small compared to the scalar pressure, we can again identify $p_{\perp}$ with $p$ for the last term.

\subsection{Pressure anisotropy}

The pressure anisotropy for a species "s" $\left(3 / 2 \pi_{\| s}\right)$ can been formulated in the fluid framework as a function of plasma flow $\left(\mathbf{V}_{\mathbf{s}}\right)$ and heat flux $\left(\mathbf{q}_{\mathbf{s}}\right)$ :

$$
\frac{3}{2} \pi_{\|, s}=-\rho \alpha_{s} C\left\{\mu_{1}^{s} \mathcal{F}\left(\mathbf{V}_{\mathbf{s}}\right)+\mu_{2}^{s} \frac{2}{5 p_{s}} \mathcal{F}\left(\mathbf{q}_{\mathbf{s}}\right)\right\}
$$

Two equivalent forms have been proposed for the operator $\mathcal{F}$ Grimm and Johnson (1972); Callen (2010):

$$
\begin{aligned}
\mathcal{F}(\mathbf{W}) & =\nabla_{\|} W_{\|}-\mathbf{W} \cdot \kappa-\frac{1}{3} \nabla \cdot \mathbf{W} \\
\mathcal{F}(\mathbf{W}) & =\mathbf{W} \cdot \nabla \ln B+\frac{\mathbf{b}}{B} \cdot \nabla \times(\mathbf{W} \times \mathbf{B})+\frac{2}{3} \nabla \cdot \mathbf{W}
\end{aligned}
$$

For numerical stability, we will show later that the first one is more appropriate for the momentum equation.

Assuming incompressible flows $\left(\nabla \cdot \mathbf{V}_{\mathbf{s}}=0\right.$ and $\left.\nabla \cdot \mathbf{q}_{\mathbf{s}}=0\right)$, we obtain the two equivalent forms for the heat flow contribution:

$$
\begin{aligned}
\frac{2}{5 p_{s}} \mathcal{F}\left(\mathbf{q}_{\mathbf{s}}\right) & =\frac{u_{\| 2, s}}{B^{2}} \mathbf{B} \cdot \nabla \ln B-\mathbf{V}_{\mathbf{T s}}^{*} \cdot\left(\kappa+\nabla \ln p_{s}\right)-\nabla \cdot \mathbf{V}_{\mathbf{T s}}^{*} \\
\frac{2}{5 p_{s}} \mathcal{F}\left(\mathbf{q}_{\mathbf{s}}\right) & =\left(\mathbf{V}_{\mathbf{T s}}^{*}+\frac{u_{\| 2, s}}{B^{2}} \mathbf{B}\right) \cdot \nabla \ln B+\frac{\mathbf{b}}{B} \cdot \nabla \times\left(\mathbf{V}_{\mathbf{T s}}^{*} \times \mathbf{B}\right)-\mathbf{V}_{\mathbf{T s}}^{*} \cdot \nabla \ln p_{s}
\end{aligned}
$$

Finally, this gives the two equivalent expressions for the pressure anisotropy

$$
\begin{aligned}
\frac{3}{2} \pi_{\|, s}= & -\rho \alpha_{s} \mu_{s} C\left\{\nabla_{\|} V_{\| s}-\left(\mathbf{V}_{\mathbf{s}}+k_{s} \mathbf{V}_{\mathbf{T s}}^{*}\right) \cdot \kappa\right. \\
& \left.-k_{s}\left(\nabla \cdot \mathbf{V}_{\mathbf{T} \mathbf{s}}^{*}+\mathbf{V}_{\mathbf{T s}}^{*} \cdot \nabla \ln p_{s}-\frac{u_{\| 2, s}}{B^{2}} \mathbf{B} \cdot \nabla \ln B\right)\right\} \\
\frac{3}{2} \pi_{\|, s}= & -\rho \alpha_{s} \mu_{s} C\left\{\left[\mathbf{V}_{\mathbf{s}}+k_{s}\left(\mathbf{V}_{\mathbf{T s}}^{*}+\frac{\left.\left.u_{\| 2, s} \mathbf{B}\right)\right] \cdot \nabla \ln B}{B^{2}}\right)\right.\right. \\
& \left.+\frac{\mathbf{b}}{B} \cdot\left[\nabla \times\left(\mathbf{V}_{\mathbf{s}} \times \mathbf{B}\right)+k_{s} \nabla \times\left(\mathbf{V}_{\mathbf{T s}}^{*} \times \mathbf{B}\right)\right]-k_{s} \mathbf{V}_{\mathbf{T s}}^{*} \cdot \nabla \ln p_{s}\right\}
\end{aligned}
$$

where we adopt the notations $\mu_{s} \equiv \mu_{1}^{s}$ and $k_{s} \equiv \mu_{2}^{s} / \mu_{1}^{s}$. We use the first formulation for the momentum equation, with $\mathbf{V}_{\mathbf{i}}=\mathbf{V}+\mathbf{V}_{\mathbf{i}}^{*}$, because the second one can introduce spurious instabilities, as shown in the following. But we use the second formulation for Ohm's law, with $\mathbf{V}_{\mathbf{e}}=\mathbf{V}+\mathbf{V}_{\mathbf{e}}^{*}-d_{i} / \rho \mathbf{J}_{\|}$, because it avoids implementing the parallel derivative of the current.

\subsection{Flow stability}

We point here the question of numerical stability for the momentum equation. For simplicity, we consider simplified pressure anisotropy, neglecting heat flows. This gives

$$
\frac{3}{2} \pi_{\|, i}=-\rho \mu_{i} C\left\{\nabla_{\|} V_{\|}-\mathbf{V} \cdot \kappa\right\}
$$


The projection of the momentum equation on the curvature and on the magnetic field gives

$$
\begin{aligned}
\rho \frac{D(\mathbf{V} \cdot \kappa)}{D t} & =\rho \mu_{i} C\left(\nabla_{\|} V_{\|}-\mathbf{V} \cdot \kappa\right) \kappa^{2}+\cdots \\
\rho \frac{D V_{\|}}{D t} & =\frac{2}{3} \nabla_{\|}\left[\rho \mu_{i} C\left(\nabla_{\|} V_{\|}-\mathbf{V} \cdot \kappa\right)\right]+\cdots
\end{aligned}
$$

with $D / D t \equiv\left(\partial_{t}+\mathbf{V} \cdot \nabla\right)$. This shows that the perpendicular flow is forced to its neoclassical value by a friction $\mu_{i} C \kappa^{2}$, while the parallel flow is damped by a parallel diffusion coefficient $\frac{2}{3} \mu_{i} C$.

Using the second formulation

$$
\frac{3}{2} \pi_{\|, i}=-\rho \mu_{i} C\{\mathbf{V} \cdot \nabla \ln B\}
$$

would lead to

$$
\rho \frac{D(\mathbf{V} \cdot \kappa)}{D t}=\rho \mu_{i} C(\mathbf{V} \cdot \kappa) \kappa^{2}+\cdots
$$

using $\kappa \approx \nabla_{\perp} \ln B$, which was observed to lead to spurious instabilities when the friction coefficient $\mu_{i}$ was too strong.

\section{Application}

\subsection{Equilibrium}

For the purpose of illustrating the implementation of the neoclassical model, we performed nonlinear simulations for a linearly unstable tearing mode. We set up a tokamak magnetic equilibrium with circular cross-section and inverse aspect ratio $\epsilon=0.3$, using the equilibrium code CHEASE Lütjens et al. (1996). The pressure profile is given by $\partial_{\psi} p \propto(1-\psi)$ and the current density profile by $I^{*} \propto(1-\psi)^{2}$ (see Lütjens et al. (1996) for the definition of $\left.I^{*}\right)$. At magnetic axis $\left(R_{0}=2.4 \mathrm{~m}\right)$, the magnetic field is $B_{0}=3 T$, the ion density $n_{i}(0)=2 \times 10^{19} \mathrm{~m}^{-3}$, electron temperature $T_{e}(0)=3910 \mathrm{eV}$, and $\tau \equiv T_{i} / T_{e}=1$, these quantities being chosen so that they are consistent with the CHEASE equilibrium : $p(0)=e n_{i}(0) T_{e}(0)[1+\tau]$. The position of $q=2$ is prescribed at $\sqrt{\psi}=0.5$, which corresponds to $x \equiv \sqrt{\Phi} \approx 0.34$ and the magnetic shear there is $s=0.585$. The density profile is prescribed in the following analytical form

$$
\rho(\psi)=\frac{1-d_{1} \psi^{d_{2}}}{1+d_{3} \psi^{d_{4}}}
$$

with $\left(d_{1}, d_{2}, d_{3}, d_{4}\right)=(0.32,1,0.1,4)$. The main plasma profiles are shown in figure 2 .

This analytical equilibrium, designed for the study of the single tearing mode $(m=2, n=1)$, is characterized by $\beta_{p}=1.12, \beta_{t}=0.15 \%, \beta_{N}=0.84$ and $l_{i}=1.58$, and a Lundquist number at magnetic axis $S_{0}^{\text {real }}=\eta^{-1}=8 \times 10^{8}$. The ion skin depth is $d_{i}=0.1$. The neoclassical equilibrium is established in a few hundred of Alfvén times, while the tearing mode is still at the noise level. During this period of time, the current source, restricted to its toroidal component, evolves so as to compensate for the variation of the bootstrap current, i.e. $\mathbf{J}_{\mathbf{C D}}(t)=\left[\mathbf{J}_{t=0}-\left\langle\mathbf{J}_{\mathbf{b s} n=0}(t)\right\rangle\right]_{\varphi}$. In the period that follows, the current source can be chosen to be kept fixed, or to continue evolving (thus compensating for the loss of bootstrap current due to the evolution of plasma equilibrium after the growth of a tearing mode for example). The bootstrap current represents about $29 \%$ of the total plasma current (figure 3 , left plot). The electron contribution to the total neoclassical stress tensor in the momentum equation is small, and slightly increases the bootstrap fraction from $29 \%$ to $30 \%$. In the simulations presented hereafter, this contribution is neglected. The comparison of the computed bootstrap current with the Sauter-Angioni evaluation Sauter et al. $(1999,2002)$ is satisfactory. Note that we damp the neoclassical terms in the very core and at the edge to reduce the discretization needs, using Lorentzian-like functions of the type $y^{n} /\left(y_{0}^{n}+y^{n}\right)$ with $y=\sqrt{\psi}$ in the core and $y=(1-\sqrt{\psi})$ in the edge, $n=6$ and $y_{0}=0.05$.

The role of parallel heat flux can be quantified by letting the equilibrium evolve without their contribution. We then find a much lower bootstrap current density, and a total contribution to the plasma current that falls from $29 \%$ to about $10 \%$ (see figure 3, middle plot). The large contribution of parallel 


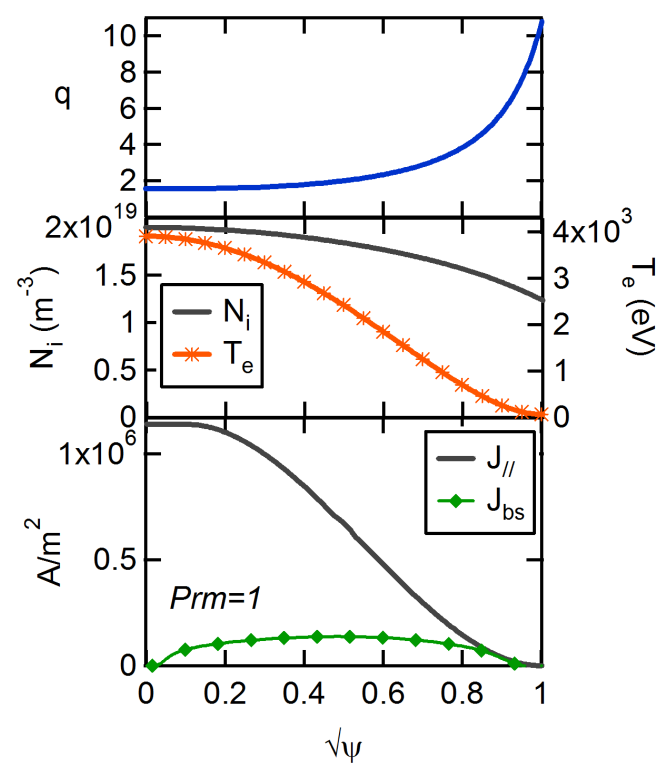

Figure 2: Safety factor profile (top), ion density and electron temperature profiles (middle), total current density and bootstrap current density profiles (bottom).

heat flows tends to decrease at larger collisionality, as shown on the right plot for a density that is three times larger (the temperature is reduced accordingly to maintain the total pressure constant). Even then however, they contribute to more than half of the total bootstrap current.

Finally, the variation of the flux averaged bootstrap current due to plasma viscosity (measured here by the magnetic Prandtl number Prm) is shown in figure 4. The departure from the analytical formulae Sauter et al. (1999, 2002) increases as Prm impacts more significantly the equilibrium plasma flow and deviates it from the neoclassical drive.

\subsection{Nonlinear simulation of a Neoclassical Tearing Mode}

We will now focus on the nonlinear aspects, i.e. the dynamics towards saturation, the structure of the perturbations and the impact of the bootstrap fraction. In these simulations, we choose to evolve the current density source so as to compensate for the variation of the averaged bootstrap current density : $J_{C D}=\left[J_{t=0}-\left\langle J_{b s}(t)\right\rangle\right]_{\varphi}$. This simplifies the interpretation of the results, since the resonant surface $q=2$ will not move due to the loss of the bootstrap current density. The situation is then equivalent to a Rutherford-like formulation where the $\Delta^{\prime}$ term is not connected to the bootstrap term. We first start by investigating the role of the mode spectrum, by including an increasing number of toroidal modes. We then evidence the role of neoclassical forces, by looking at the impact of the bootstrap current contribution on the island saturation.

For these simulations, we choose $S_{0}=10^{7}$, i.e. a resistivity that is about 80 times larger than the real one, and a magnetic Prandtl number Prm $\equiv \nu / \eta=10$. We also take $\chi_{\|} / \chi_{\perp}=10^{8}, \chi_{\perp} / \eta=150$, and $D_{\perp}=2 / 3 \chi_{\perp}$. The radial discretization is of 510 points, and we consider a maximum physical toroidal mode number ranging from 2 to 8 , taking into account aliasing constraints Press et al. (2007). In the poloidal direction, poloidal mode numbers in the range $\left[\max \left(0, n-m_{\text {inf }}\right), n+m_{\text {sup }}\right]$ are described, with $n \in\left[0, n_{\max }\right], m_{\text {inf }}=12$ and $m_{\text {sup }}=23$. 


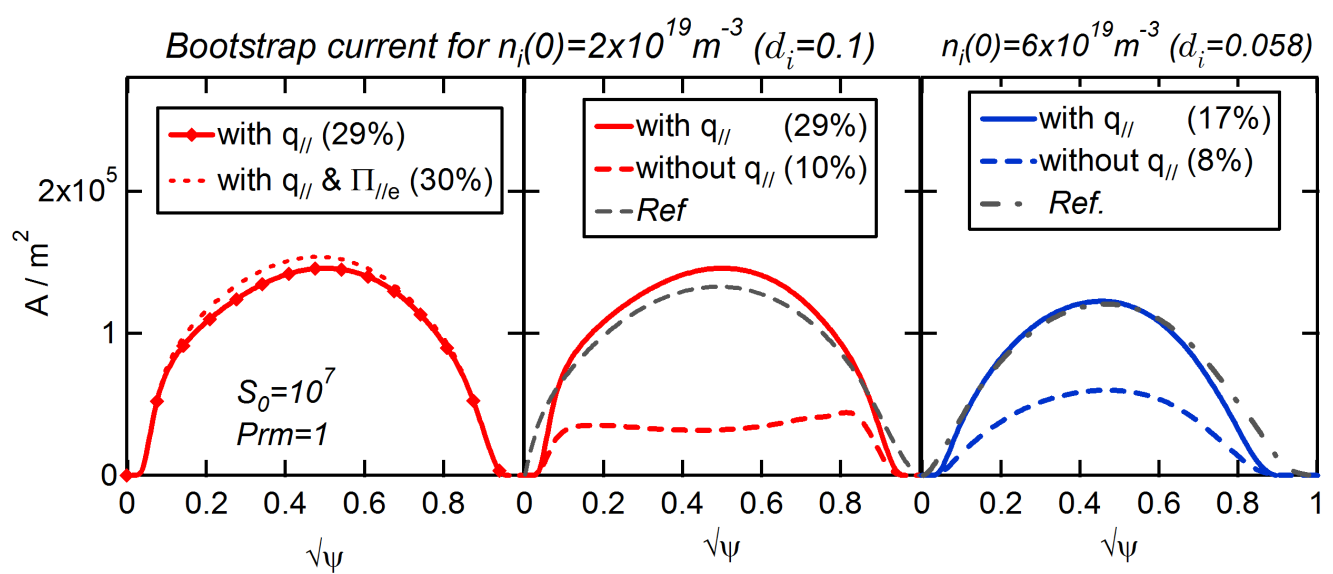

Figure 3: Equilibrium bootstrap current and analytical formulae from Sauter et al. (1999, 2002) (left). Equilibrium bootstrap current with and without the parallel heat flow contribution, and with the electron contribution in the momentum equation, for the reference low density case (middle), and for larger density (right). The numbers in parenthesis indicate the fraction of total bootstrap current.

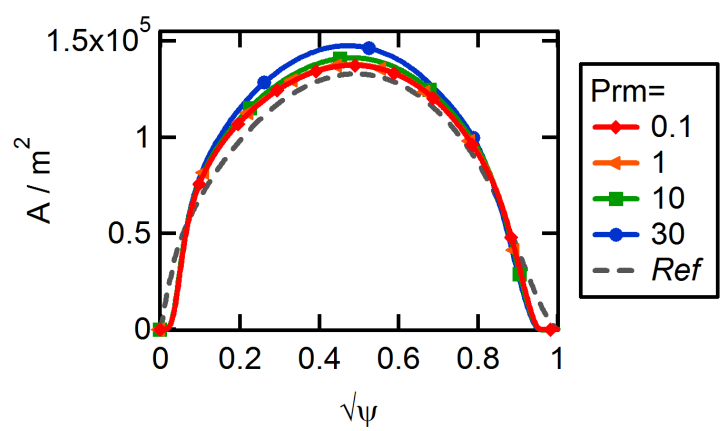

Figure 4: Equilibrium bootstrap current for different magnetic Prandtl numbers $(\operatorname{Prm}=\nu / \eta)$, compared with the analytical formulae from Sauter et al. (1999, 2002). 


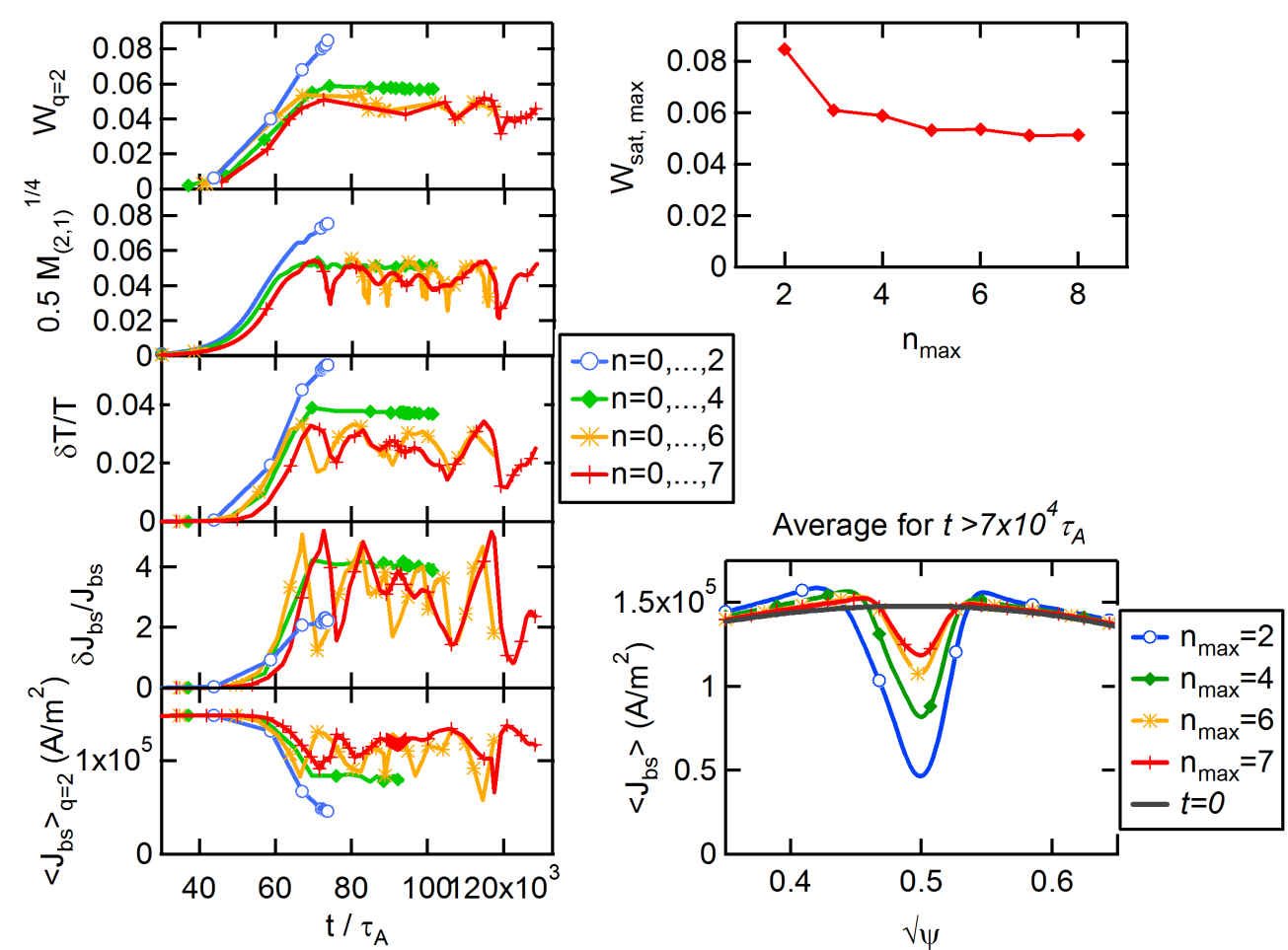

Figure 5: Summary on the influence of the mode spectrum. Left from top to bottom: time evolution of the island width on $q=2$ from Poincaré maps ; of $0.5 M_{(2,1)}^{1 / 4}$, where $M_{(2,1)}$ is the magnetic energy of the $(2,1)$ mode ; maximum relative temperature fluctuation $\delta T / T$; maximum relative bootstrap current fluctuation $\delta J_{b s} / J_{b s}$; flux averaged bootstrap current at $q=2$. Top right: maximum saturation level of the island on $q=2$ (determined from Poincaré plots) as a function of the maximum toroidal mode number $n_{\max }$ considered in the simulation. Bottom right: mean profile at saturation $\left(t>7 \times 10^{4} \tau_{A}\right)$ of the flux averaged bootstrap current for $n_{\max }=2,4,6$ and 7 .

\subsubsection{Nonlinear dynamics of the Neoclassical Tearing Mode}

We first investigate the saturation of the Neoclassical Tearing Mode with its nominal bootstrap fraction (i.e. $f_{b s}=1$ ), for different spectra of modes. In terms of physical modes, we consider a spectrum $n=0, \ldots, n_{\max }$ with $n_{\max }$ ranging from 2 to 8 . A summary of the results of numerical simulations is displayed in figure 5, where we show the evolution of a quantity that is generally representative of the $(2,1)$ island width, $0.5 M_{(2,1)}^{1 / 4}$, where $M_{(2,1)}$ is the magnetic energy of the $(2,1)$ mode, the evolution of the relative temperature and relative bootstrap perturbations in the island region, as well the averaged bootstrap current density at $\sqrt{\psi}=0.5$. The relative bootstrap perturbation is defined as the maximum difference between the extrema of the $n \neq 0$ component of the bootstrap current (equation 19), divided by its mean value. The figure also shows the maximum island width (as measured from Poincaré maps) as a function of $n_{\max }$, as well as the mean profile of the averaged bootstrap current in the island region at saturation.

For the lowest resolution $(n=0, \ldots, 2)$, the island grows above $8 \%$ of the minor radius (it has not saturated yet at the end of the simulation), and the averaged bootstrap current is strongly decreased (due to the reduction of temperature and density gradients) in the island region. For $n_{\max } \geq 3$, the saturation is completed at a lower island size, in the range $5-6 \%$ of the minor radius, and the impact on the averaged bootstrap current is also reduced.

Interestingly, when the resolution is large enough, the nonlinear saturation is not steady, but it is 


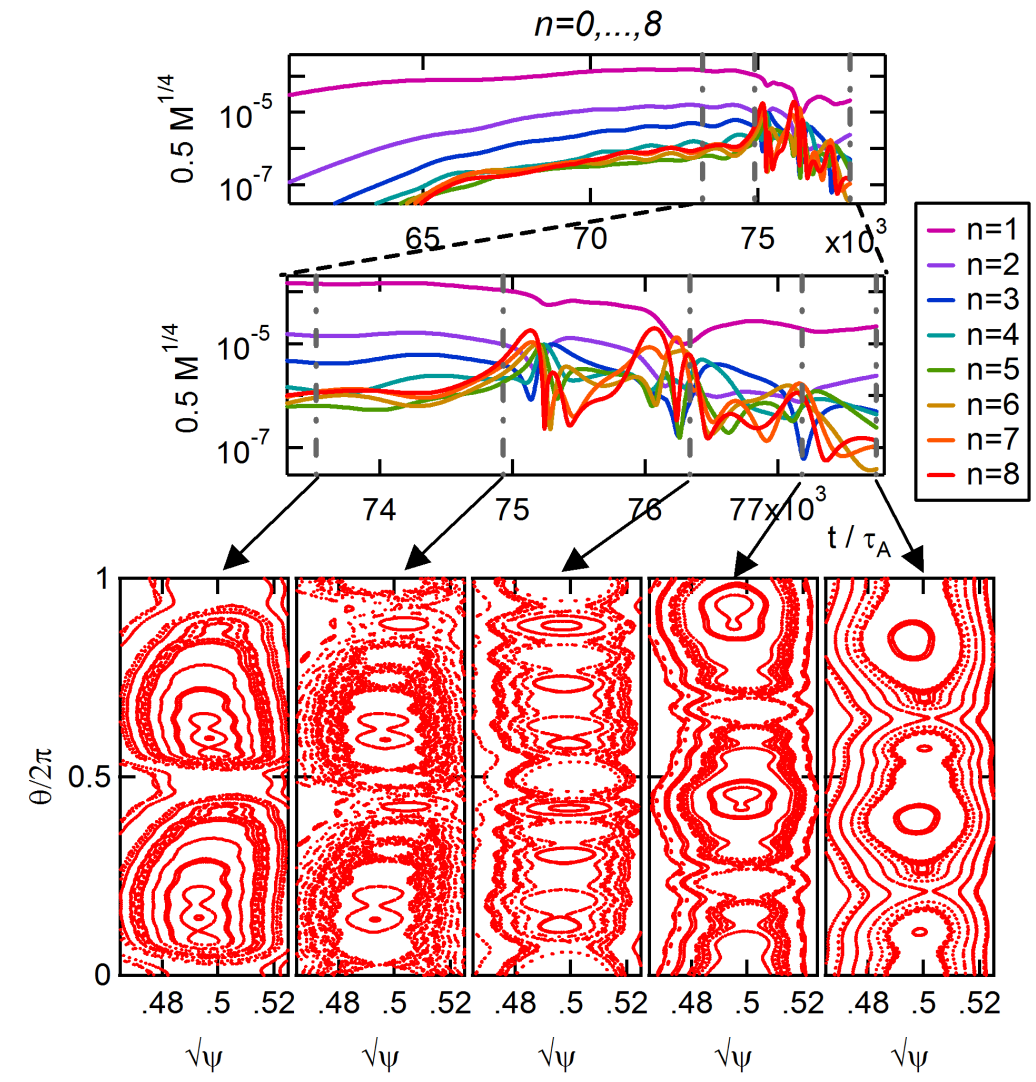

Figure 6: Evolution of $M_{n}^{1 / 4}$ with $M_{n}$ the magnetic energy of toroidal mode number $n$, and Poincaré maps at selected times during the first burst of small scale modes after reaching saturation $\left(n_{\max }=8\right)$.

accompanied by bursts of energy originating from the smallest structures. The level of fluctuations varies a lot during these bursts, but the island size measured by the maximum radial extent of the separatrix is less sensitive. In order to give a better idea of this phenomenon, we show in figure 6 the evolution of mode energies and island structure during such a burst, for the case $n_{\max }=8$. The Poincaré maps first show that the island tends to have a droplet shape when small scale structures are well below the size of the dominant $n=1$ component (first and last maps). The mode is rotating in the electron direction (upwards in these figures) relative to the plasma frame (see section 3.2.5). The burst of small scale structures gives rise to a fragmentation of the island into a chain of small islands having an effective structure corresponding to the highest $n$ 's. Note that during this phase, the quantity $0.5 M_{(2,1)}^{1 / 4}$ underestimates the maximum distance between separatrices (because the island chain has $n>1$ ), and is no longer representative of the island width on $q=2$.

This phenomenon is reminiscent of current sheet instabilities observed with the standard resistive MHD model Waelbroeck (1993); Loureiro et al. (2005). In such a case, however, the critical island size $W_{c}$ above which the current sheet becomes unstable is relatively large, with a threshold that scales as $a \Delta^{\prime} W_{c}>C(\eta)$ with $C(\eta)>8.2$ following Loureiro et al. (2005). In our case where the cylindrical $a \Delta^{\prime} \approx 27$, the current sheet would be unstable for $W>0.3$, i.e. much above the actual size of our $(2,1)$ island. Such a disagreement is not surprising because the issue of current sheet instabilities is usually encountered in large $\Delta^{\prime}$ reconnection problems, such as for the internal kink, space plasmas, or for the forced reconnection problem Ishii et al. (2007); Comisso et al. (2015). However, the constant- $\psi$ hypothesis that is usually assumed in tearing mode theory becomes invalid as diamagnetic effects are increased Yu et al. (2003), so that the current sheet instability criterion mentioned above is outside its domain of validity for drift-tearing modes. Secondary island formation Lazzaro et al. (2010) or island fluctuations 

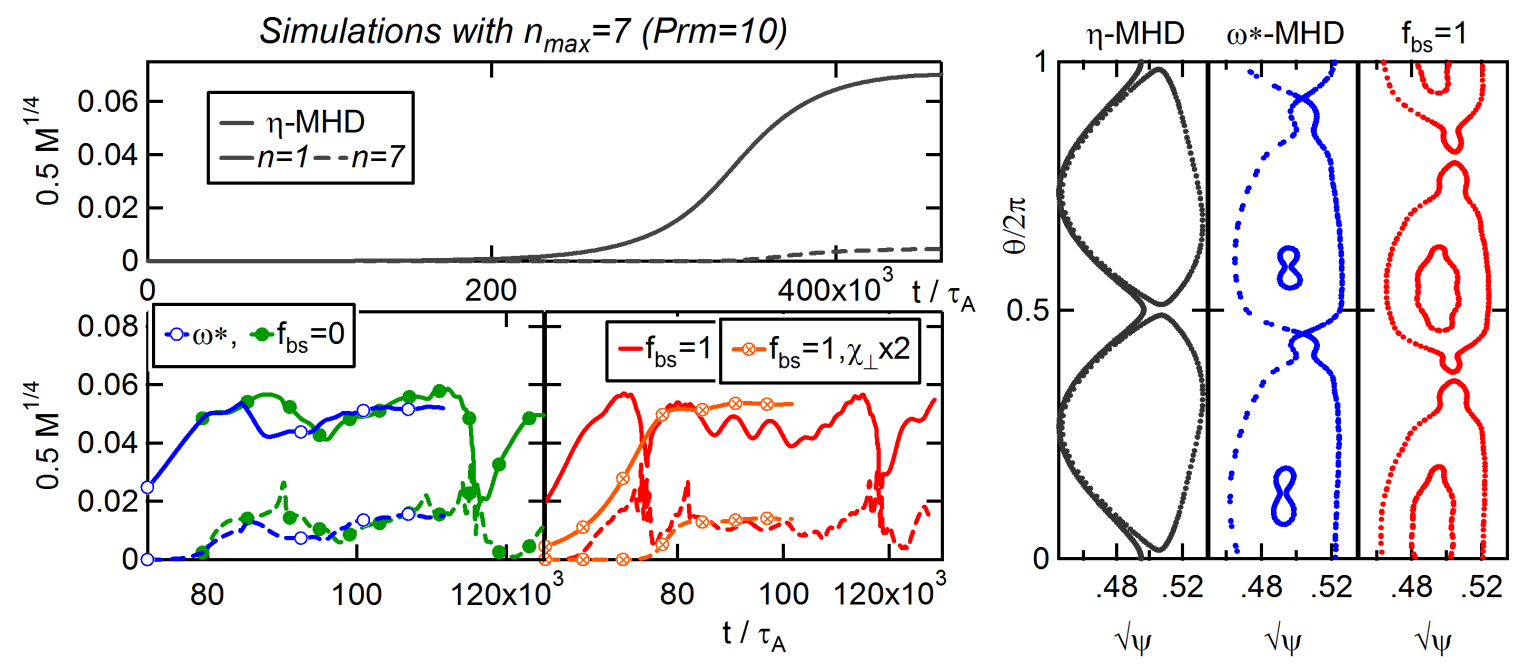

Figure 7: Evolution of $M^{1 / 4}$ with $M$ the magnetic energy of toroidal mode numbers $n=1$ (full line) and $n=7$ (dashed line) for simulations with the resistive MHD model ( $\eta$-MHD), the drift-tearing model $\left(\omega^{*}\right)$, including the ion neoclassical friction $\left(f_{b s}=0\right)$, with the full neoclassical model with $f_{b s}=1$ and a larger $\chi_{\perp}$ and $D_{\perp}$, and for the reference case with $f_{b s}=1$. The shapes of the island at the end of simulations with $\eta$-MHD, $\omega^{*}$-MHD and with the full neoclassical model, are shown in the right panel. For all these cases, $n_{\max }=7$.

Yu (2010) have been observed with advanced physical models, comparable with the one presented here, in the simulation of drift-tearing instabilities with neoclassical effects.

In order to identify what drives these bursts, we have performed simulations at $n_{\max }=7$ with different MHD models, starting with the drift-tearing model ( $\omega^{*}$-MHD), and adding (after $t \approx 80 \times 10^{3}$ ) ion neoclassical friction $\left(f_{b s}=0\right)$. In figure 7 , the evolution of $M^{1 / 4}$ is shown for $n=1$ and $n=n_{\max }$ for these different cases, as well as for the reference case with $f_{b s}=1$ and for the resistive MHD model $(\eta$-MHD). The dynamics of the tearing mode is fastened by diamagnetic rotations (as reported in several works Yu et al. (2003); Maget et al. (2014)), and the level of the highest $n$-harmonics is much above that of the $\eta$-MHD case for all models covering drift physics. Bursts are visible as soon as ion neoclassical friction is included: they are therefore strongly connected with neoclassical forces, and are amplified by the bootstrap current contribution. The shapes of the island at saturation are compared on the right plots of figure 7, showing that the droplet shape emerges with the drift model, and is therefore not specific to the neoclassical model discussed here.

Dissipation mechanisms are able to damp these bursts. We find that this is the case when the perpendicular transport is increased (in figure 7, we show a simulation where perpendicular transport coefficients $\chi_{\perp}$ and $D_{\perp}$ are increased by a factor of 2 , while $\chi_{\|}$is left unchanged). We will show in the next section (3.2.2) that plasma viscosity has a similar mitigating effect.

\subsubsection{Influence of plasma viscosity}

The way bootstrap current arises in the plasma means that plasma viscosity might influence not only its amplitude at equilibrium but also its relation to perturbed fields. The island dynamics should therefore respond to a change of the magnetic Prandtl number Prm, as shown in Konovalov et al. (2005); Mikhailovskii et al. (2005). In order to verify this, we restart the simulation with $f_{b s}=1$ and $n_{\max }=7$, that exhibits for Prm $=10$ an unsteady dynamics with frequent bursts of island breaking into small secondary structures. In figure 8 we show the evolution of the island size on $q=2$ and of the magnetic energy $M_{n}^{1 / 4}$ with $n=1$ and $n=n_{\max }=7$, for $\operatorname{Prm}=2,10,20$ and 50. At the time of the restart, a burst is growing, with a decay of the $n=1$ magnetic energy in favour of higher toroidal mode numbers. 

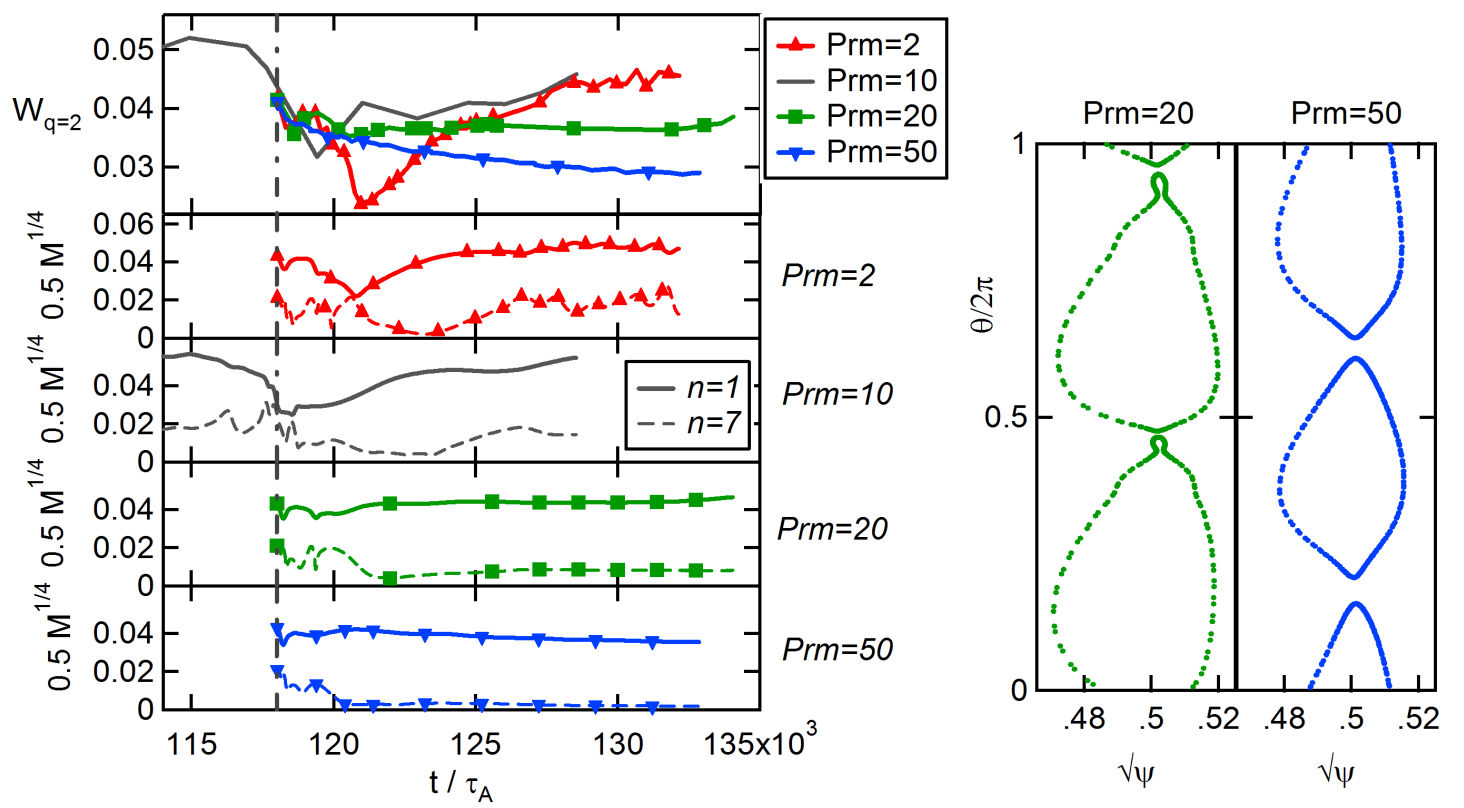

Figure 8: Island size on $q=2$ (top), and $M_{n}^{1 / 4}$ for $n=1$ and $n=n_{\max }$ for Prm=2, 10, 20 and 50 . Right plots: island separatrix at the end of the simulation for Prm $=20$ and $\operatorname{Prm}=50$.

At $\operatorname{Prm}=2$, the relative contribution of the highest $n$ is large during the burst but for Prm $\geq 20$, the burst simply disappears, replaced by a situation where high $n$ modes have a much lower importance. The shape of the island becomes also more regular as Prm is increased, although its droplet shape remains. The saturated island size becomes steady and decays with Prm. We have evidence from another study with the same equilibrium that in pure resistive MHD the island size would rather increase with Prm in this range Maget et al. (2016). The reason for the present observation is therefore to be found in the drift-neoclassical physics that is used.

\subsubsection{Neoclassical effect on island saturation}

We now evidence the role of the neoclassical contribution to the island saturation for the case $n_{\max }=4$. This resolution has been chosen because it correctly describes the saturation size without triggering the current sheet instability that slows down simulations at larger toroidal mode numbers. For this purpose, we restart the simulation $f_{b s}=1$ with different values of $f_{b s}$. Note that since the current density source always adapts in time with the actual averaged bootstrap current, there is no shift in the position of the resonant surface when performing this scan. The evolution of the island size, poloidal beta, and relative temperature perturbation is shown in figure 9 .

As expected, the island size increases monotonically with the bootstrap current amplification, as well as the peak relative temperature and bootstrap perturbations, while the poloidal beta $\left(\beta_{p}\right)$ varies as the inverse of the island size. The non-steady nature of the NTM saturation is enhanced as the bootstrap drive is increased. It is clear from these results that our $(2,1)$ island is dominated by classical effects, so that the introduction of the neoclassical drive changes only marginally the saturated size. The dynamics of the mode can be analysed with the simplified formalism of the Rutherford equation Rutherford (1973) that writes

$$
d W / d \tau_{\eta}=a \Delta^{\prime}-\alpha W-6.35 \frac{D_{R}}{\sqrt{W^{2}+W_{D}^{2}}}+6.35 f_{b s} \bar{J}_{b s} \frac{q}{s} \frac{W}{W^{2}+W_{b s}^{2}}
$$

Here $W$ is the island width normalized to the minor radius and $\tau_{\eta} \equiv 1.22 t / S$. The first term $\left(a \Delta^{\prime}\right)$ describes the classical tearing index that drives the growth of tearing modes in resistive MHD Furth 

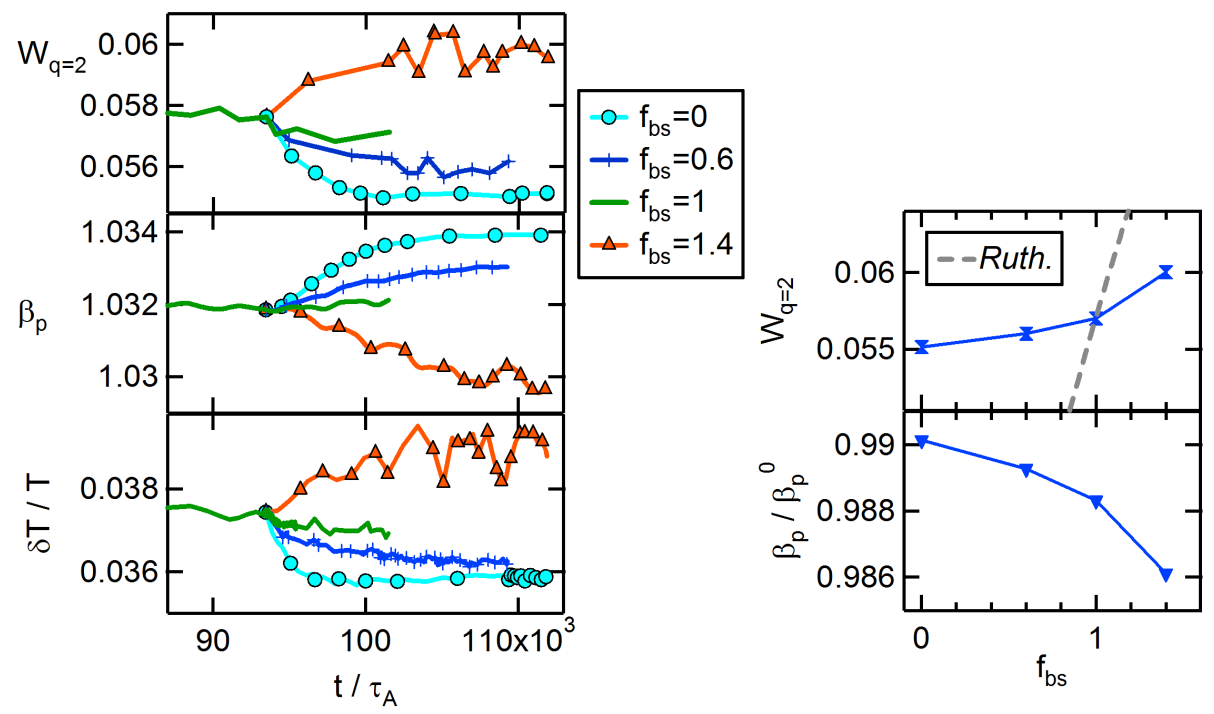

Figure 9: Left from top to bottom: Evolution of the $q=2$ island size, poloidal beta, relative temperature perturbation, for different amplifications of the bootstrap current. Right: saturated island size and poloidal beta reduction as a function of the bootstrap current amplification.

et al. (1963), the second term describes the simplest form of classical nonlinear saturation Escande and Ottaviani (2004); Militello and Porcelli (2004). The third term describes curvature stabilisation Lütjens et al. (2001) with $D_{R}$ the resistive interchange index Glasser et al. (1975), and the fourth covers the bootstrap drive Fitzpatrick (1995) with $f_{b s}$ the artificial amplification of the bootstrap current and $\bar{J}_{b s}=$ $\left(\mu_{0} R_{0} / B_{0}\right) J_{b s}$ the normalized bootstrap current. Due to the role of transport in the nonlinear behaviour of pressure-related quantities, the corresponding characteristic width $W_{\chi}$ is involved in both curvature and bootstrap terms, with $W_{D}=0.81 W_{\chi}$ and $W_{b s}=1.8 W_{\chi}$, and $W_{\chi}=2 \sqrt{2}\left(\chi_{\perp} / \chi_{\|}\right)^{1 / 4} \sqrt{x /(\epsilon n s)}$, with $x=\sqrt{\Phi}$ the radial co-ordinate with $\Phi$ the normalized toroidal magnetic flux, $\epsilon=a / R_{0}$ the inverse aspect ratio, $n$ the toroidal mode number, $s=d(\log q) / d(\log x)$ the magnetic shear and $q$ the safety factor. In our example, we have $W_{\chi} \approx 0.037, W_{D} \approx 0.030, W_{b s} \approx 0.067$ and $D_{R} \approx 0.07$. The classical drive plays an important role because the parameter $\alpha$ is large : with $a \Delta^{\prime} \approx 27$ and a saturation around $5.5 \%$ at $f_{b s}=1$, we find $\alpha \approx 770^{1}$. Given these numbers, with $\overline{J_{b s}} \approx 0.149$ at $q=2$, one can compare the predictions of the Rutherford equation (36) with nonlinear simulations in terms of saturated island width. The result is also shown in figure 9. The impact of the bootstrap current predicted by the Rutherford model is found to be far too large. A similar observation was already reported with a simplified bootstrap model in Lütjens and Luciani (2005). In order to recover the simulation results, with $\alpha$ a free parameter, the contribution of the bootstrap term should be reduced to about $5 \%$ of its value, or the transport with $W_{\chi}$ increased by a factor 5. The Rutherford equation (36) seems therefore unable to correctly describe the island saturation in the present case. However, by using the scaling factor $f_{b s}$, we have varied independently parameters that in reality are connected. It is possible that the reasonable qualitative agreement between experimental measurements of NTM dynamics and simple Rutherford models like equation (36) can only be obtained for some particular coherent parameter scans (for example a scan in $\beta$ that will increase at the same time $\omega^{*}, J_{b s}$ and $\left.D_{R}\right)$, as was found in $\mathrm{Yu}(2010)$.

\footnotetext{
${ }^{1}$ A simplified evaluation like in Escande and Ottaviani (2004); Militello and Porcelli (2004) would predict $\alpha \approx 0.41$, but our situation is not in the range of validity of these theoretical works because of a large $a \Delta^{\prime}$ and non-symmetric equilibrium. We only retain here the functional dependence.
} 


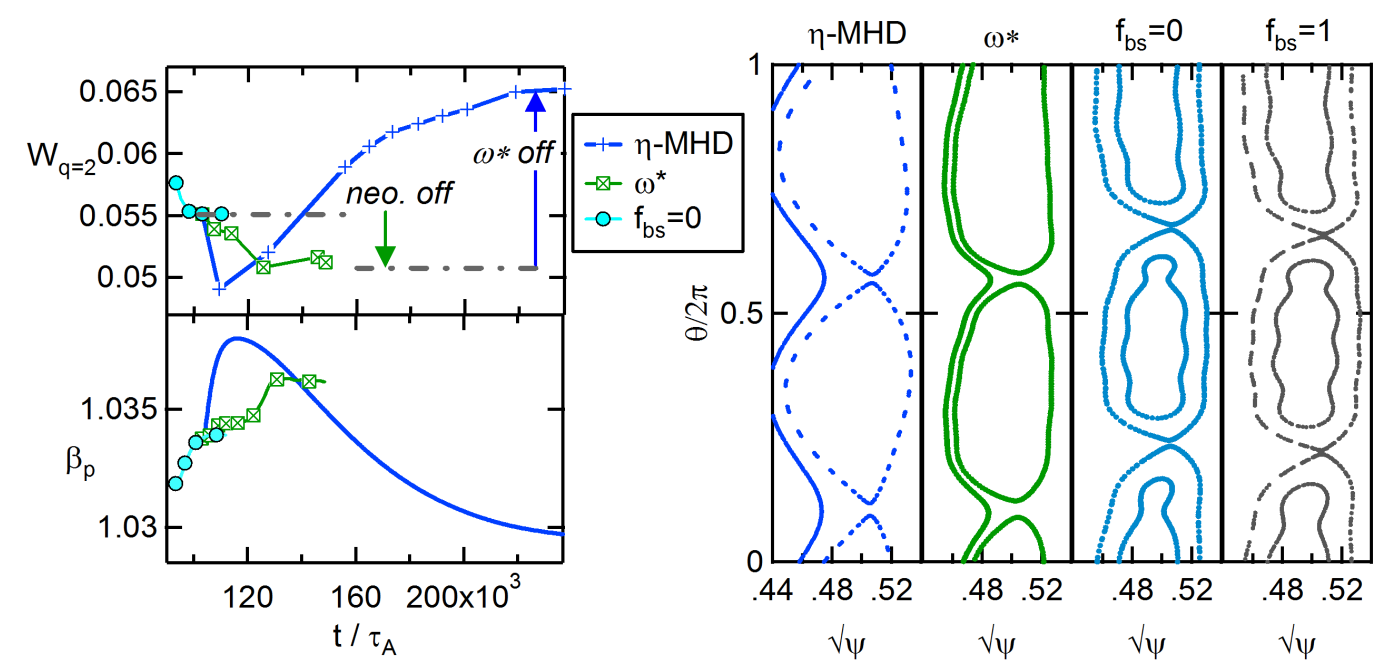

Figure 10: Left from top to bottom: Evolution of the $q=2$ island size (determined from Poincaré maps), and poloidal beta for the resistive MHD model, the drift-tearing model and the neoclassical model without bootstrap current. Right: Poincaré maps at the end of each simulations, including the case $f_{b s}=1$ for reference (here $n_{\max }=4$ ).

\subsubsection{Diamagnetic and neoclassical effects on the island properties}

Although the drive of the magnetic island is mainly classical, it would be wrong to believe that its saturation could be described with a pure resistive MHD model, even in the case where the bootstrap current contribution is cancelled $\left(f_{b s}=0\right)$ : the ion neoclassical friction as well as diamagnetic rotations have a noticeable effect on the saturated island. This is demonstrated in figure 10 where the simulation with $f_{b s}=0$ is pursued without the ion neoclassical friction (the MHD model is then the drift-tearing model), and without both neoclassical terms and diamagnetic terms (only remains the pure resistive MHD model). Removing the ion neoclassical friction reduces the saturation size, an effect that was already observed for the Double-Tearing Mode Maget et al. (2014) and that is consistent with the destabilizing effect of neoclassical friction in the linear regime Maget et al. (2013). When returning to the pure resistive MHD model $(\eta$-MHD), the island first shrinks but after this transient phase, it grows to a much larger size. This complex dynamics is to be placed in the context of previous works on the effect of diamagnetic rotations in toroidal plasmas. It was shown in Meshcheriakov et al. (2012) that increasing diamagnetic rotations increases the growth rate of the tearing mode in the first place, before a stabilizing effect can be observed. Here, we are in a range of the ion skin depth $d_{i}$ where the linear growth rate is largely increased compared to the resistive MHD case. Removing diamagnetic rotations moves the tearing mode towards a more stable situation from the point of view of its linear stability. On the other hand, the saturation of the tearing mode is reduced by diamagnetic rotations Ottaviani et al. (2004); Meshcheriakov et al. (2014). When we remove this mechanism, we logically end up with a larger island. The first instantaneous effect of island shrinking may be related to the fact that the island, placed in the new situation of $\eta$-MHD, is then far below its saturated value, thus putting forward the linear stabilizing effect.

We also plot in figure 10 the Poincaré map of field lines located close to the island separatrix at the end of each simulation. As for simulations at larger $n_{\max }$, we observe that the deformed island shape essentially originates from diamagnetic rotations (see figure 7 ). But at this resolution $\left(n_{\max }=4\right)$, the island does not have the droplet shape (it starts to appears for $n_{\max }=5$, and is clearly observable at $n_{\max }=6$ ): covering a broad spectrum of toroidal mode numbers appears therefore to be essential for describing correctly the shape of the island, although its size does not seem to be very sensitive. Note that such a deformation of the island shape could impact the efficiency of a current source to stabilize it Lazzaro and Nowak (2009); Lazzari and Westerhof (2011).

The influence of the MHD model on the island shape can also be analysed from the temperature 
and density fluctuations. We will not address this issue from a quantitative point of view: for the parameters that we have chosen, this would not only require covering a large spectrum of toroidal modes, but also performing long simulations to obtain a correct statistics on the bursts. Instead, we aim here at giving a qualitative idea of how the MHD model can impact observable quantities. For this purpose, we mimic the rotation of the plasma in front of a synthetic measurement by recording the profiles along the toroidal angle. We then compute the power spectrum $P_{S}(\sqrt{\psi}, n)=(\delta S)^{2} / \bar{S}^{2}$ of the signal $S(\sqrt{\psi}, t)(\bar{S}$ is the $n=0$ component of $S$, that would correspond to the time averaged value in a real experiment). This gives a radial profile of the fluctuating amplitude for the different toroidal mode numbers that are represented in the simulation. In figure 11 we show on the left side the radial maximum of the power spectrum as a function of the toroidal harmonic, for temperature and density fluctuations, normalized to the power spectrum of the $n=1$ toroidal harmonic. We find again, from this other perspective, that diamagnetic rotations are the essential ingredient changing the nonlinear mode spectrum by amplifying the contribution of high- $n$ modes, while neoclassical friction tends to further reinforce this trend. The decay of temperature fluctuations with the toroidal mode number can be fitted with a power law $(\delta T / T)^{2} \sim n^{-\alpha}$, with $\alpha \approx 2.3$ for the resistive MHD case, and $\alpha \approx 1$ when diamagnetic rotations are included. A different fit must be chosen for density fluctuations. We find that an exponential law $(\delta N / N)^{2} \sim \exp (-\alpha n)$ is adapted, with $\alpha \approx 1.45$ for resistive MHD and $\alpha \approx 0.45$ with diamagnetic rotations. We note that the same fitting function is also convenient for density fluctuations caused by turbulence in real plasmas Hennequin et al. (2004).

The radial profile of $n=1$ fluctuations (figure 11, right plots) shows also some interesting features. First, we find that diamagnetic rotations reduce the radial extent of density fluctuations on the high field side (HFS) of the plasma. At the same time, the asymmetry between the high field side and low field side (LFS) is enhanced: $(\delta N / N)_{L F S} /(\delta N / N)_{H F S}$ increases from about 1.04 to about 1.5. This is the signature of a more ballooned character of the density fluctuations. Temperature fluctuations are less concerned in terms of radial profile or asymmetry between HFS and LFS, due to the large parallel equilibration. However, the relative amplitude of temperature compared to density fluctuations is increased when diamagnetic rotations are considered: the ratio $(\delta T / T)^{\max } /(\delta N / N)^{\max }$ increases from about 2.2 to 3 . Neoclassical physics do not significantly change this picture, in the absence of the sporadic bursts that will obviously alter the mean spectra at higher toroidal resolution.

Diamagnetic and neoclassical physics can therefore be measured qualitatively and quantitatively in these numerical experiments, with significant departure from the resistive MHD model predictions. Comparison with real experimental observations may provide a confirmation of their impact on the MHD signature of magnetic islands.

\subsubsection{Plasma and island rotation}

The mode pulsation is evaluated from the rotation of the X-point poloidal positions $\theta_{X}(t)$ at a fixed toroidal angle $(\varphi=0)$. In order to determine the X-point positions, we detect the poloidal angles where $\delta p$ has a maximum, in the vicinity of the resonant layer. We get therefore :

$$
m \theta_{X}(t)=\omega\left(t-t_{0}\right)
$$

with $m$ the poloidal mode number. Note that since we use here the geometrical angle, the fit can only be adjusted at a given poloidal position, with a distortion at other angles (that is negligible in our case due to the low $\beta$ ).

The plasma rotation needs to be taken into account because the important quantity is the mode rotation in the plasma frame where $V_{E}=0$. It is computed from the $n=0$ component of the plasma flow as $\omega_{E}=m \omega_{\theta}-n \omega_{\varphi}$ where

$$
\begin{aligned}
\omega_{\theta} & =2 \pi / \oint \frac{\mathcal{J} d \theta}{R V_{\theta, n=0}} \\
\omega_{\varphi} & =\oint d \theta V_{\varphi, n=0} /(2 \pi R)
\end{aligned}
$$

and $\mathcal{J} \equiv 1 / \nabla s \cdot \nabla \theta \times \nabla \varphi$ is the Jacobian of the co-ordinate system $(s \equiv \sqrt{\psi}, \theta, \varphi)$ used in XTOR. These formula are obtained by computing the period $T_{\theta, \varphi} \equiv \oint d l_{\theta, \varphi} / V_{\theta, \varphi}$ with $d l_{\theta}=\mathcal{J} d \theta / R$ and $d l_{\varphi}=R d \varphi$. 

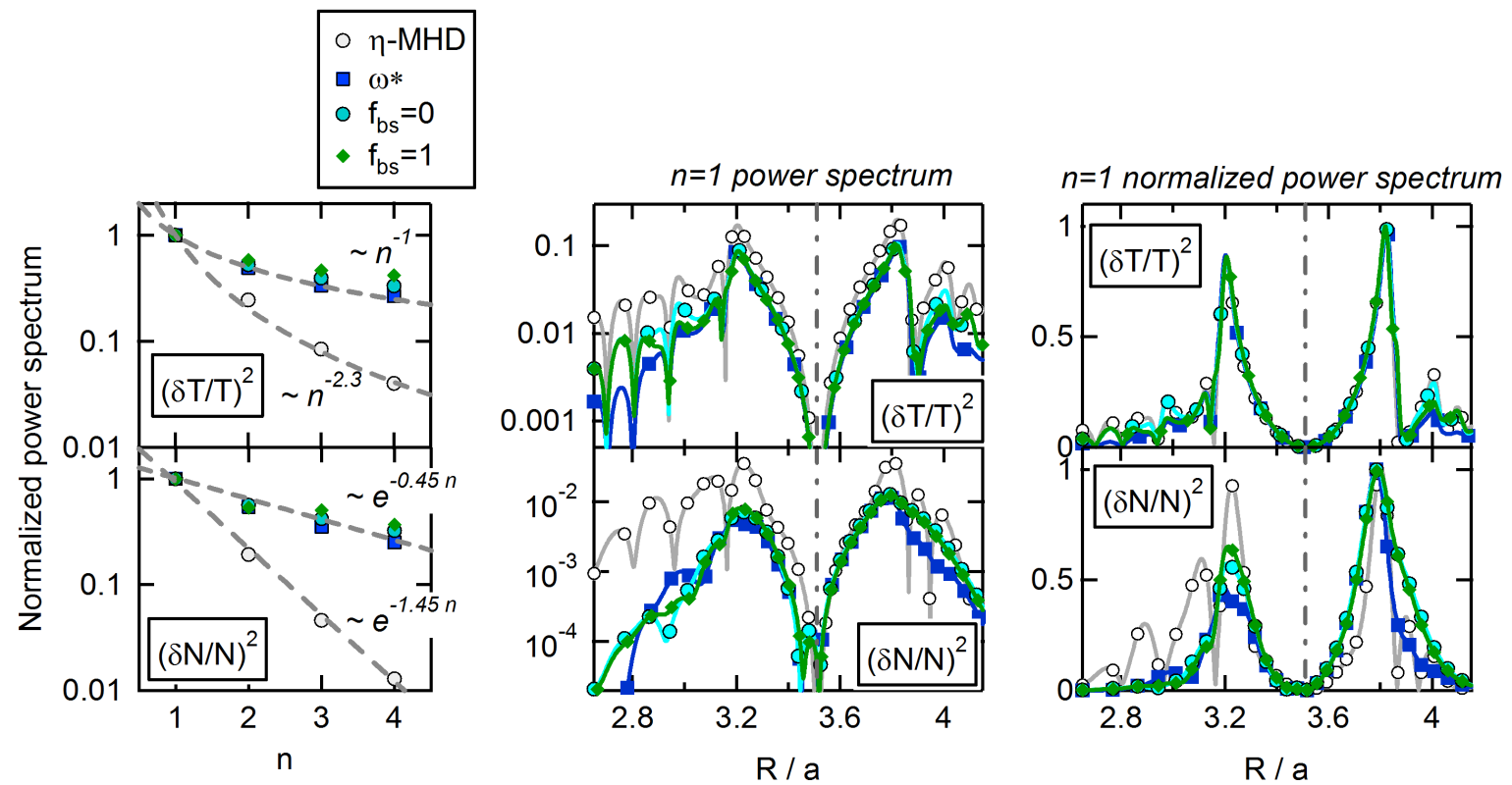

Figure 11: Power spectrum of temperature and density fluctuations for $n_{\max }=4$ : normalized to $n=1$ amplitude (left column), radial profile for $n=1$ (middle column), and normalized radial profiles for $n=1$ (right column) for the different MHD models : resistive $\left(\eta\right.$-)MHD, with diamagnetic rotations $\left(\omega^{*}\right)$, and for the neoclassical model with $f_{b s}=0$ and $f_{b s}=1$. The vertical dashed line on the middle and right plots shows the position of the magnetic axis.

The ion and electron diamagnetic pulsations are evaluated at the resonant surface $q=2$ in the same way as $\omega_{\theta}$, using $V_{i, \theta}^{*}$ and $V_{e, \theta}^{*}$ instead of $V_{\theta, n=0}$ (we have in our simulations $\omega_{i}^{*}=-\omega_{e}^{*}$ ):

$$
\omega_{e, i}^{*}=2 \pi m / \oint \frac{\mathcal{J} d \theta}{R V_{e, i, \theta}^{*}}
$$

The plasma rotation is $\omega_{E} \approx-0.9 \omega_{i}^{*}$ when considering only diamagnetic terms (figure 12). This corresponds to the theoretical value $\omega_{E}=-2 q^{2} /\left(1+2 q^{2}\right) \omega_{i}^{*}$ Nicolas $(2013)$ that is expected. With neoclassical friction, its amplitude is reduced down to $\omega_{E} \approx-0.4 \omega_{i}^{*}$. Note that this rotation is not identical to the one that is forced by neoclassical friction. Indeed, with $k_{i} \approx-1$ at the $q=2$ surface, neoclassical friction tends to force $\left(\omega_{E}-\omega_{n}^{*}\right) \approx 0$ with $\omega_{n}^{*}=-\left(\omega_{i}^{*}-\omega_{T i}^{*}\right)$ and $\omega_{T i}^{*}=k_{\theta} V_{T i \theta}^{*}$. But $\omega_{n}^{*} \approx-0.2 \omega_{i}^{*}$ so that the $\mathbf{E} \times \mathbf{B}$ flow is at an intermediate value between the diamagnetic and neoclassical drives.

The mode frequency at saturation, after deducing the $\mathbf{E} \times \mathbf{B}$ pulsation, is in the electron direction, a result that is in accordance with nonlinear computations in the cold ion limit Waelbroeck (2007); Yu (2010). With the $\omega^{*}$ model, we find $\omega / \omega_{e}^{*} \approx 0.69$ and with ion neoclassical friction (case $f_{b s}=0$ ), the pulsation is $\omega / \omega_{e}^{*} \approx 0.86$. It stays in this domain of frequency when including the bootstrap current, with $\omega / \omega_{e}^{*} \approx 0.91$ at $f_{b s}=1$.

\section{Conclusion}

In this paper we have presented a comprehensive implementation of neoclassical physics in a fluid representation that is appropriate for global MHD computations. As an illustration of the model, the nonlinear evolution of a toroidal plasma with circular cross-section, unstable to the $(2,1)$ tearing mode, is investigated. The equilibrium bootstrap current that is obtained compares well with the inviscid solution of flux averaged neoclassical theory, but it could depart from it at larger magnetic Prandtl number. The $(2,1)$ magnetic island is shown to evolve to a non-steady saturation, where it adopts a droplet shape and is 


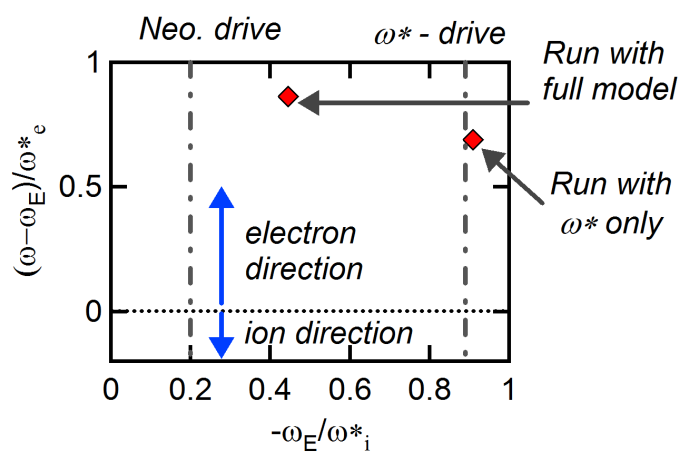

Figure 12: Island pulsation in the plasma frame normalized to the electron diamagnetic frequency at $q=2$, as a function of the plasma rotation, for the drift model $\left(\omega^{*}\right)$ and the neoclassical model.

subject to bursts of small scale structures due to secondary islands with high toroidal mode numbers that are reminiscent of current sheet instabilities. These bursts are found to originate from neoclassical forces, but their precise nature remains to be determined. Dissipative mechanisms (larger magnetic Prandtl number or larger diffusion coefficients) tend to mitigate them. The saturated state is shown to increase with the bootstrap current fraction, but much less than predicted by a generalized Rutherford equation, an observation that is reminiscent of previous works using a simplified bootstrap representation Lütjens and Luciani (2005). The comparison with MHD models of reduced complexity shows the essential role of diamagnetic rotations and neoclassical friction in the island deformation and saturation level.

The implementation of this self-consistent fluid model opens the route to the investigation of several important issues for the understanding of Neoclassical Tearing Mode physics. One particular specificity of our neoclassical model is the fact that the bootstrap current does not depend only on the perturbed pressure, as it is the case when neoclassical physics is implemented as an ad-hoc term in a resistive MHD framework, but also on the perturbed electrostatic potential. This opens a wide area for studying Neoclassical Tearing Mode physics in viscous plasmas, where theoretical approaches predict for example a strong reduction of the bootstrap perturbation as plasma viscosity increases Konovalov et al. (2005); Mikhailovskii et al. (2005). It was already shown that the freedom given to the plasma flow to depart from its neoclassical reference changes significantly the NTM threshold Mellet et al. (2013). Here we have shown, as a preliminary insight into this issue, that plasma viscosity changes the spectral content of the MHD perturbation. Another issue is the comparison of experimental signals with the modelled ones in order to investigate questions relative to the island shape or the spectral composition of Neoclassical Tearing Modes in real plasmas. Finally, the impact of the deformed island shape on tearing mode stabilization by an external current drive can be evaluated thanks to the implementation of an RF control module Février et al. (2016).

\section{Aknowledgments}

This work has been carried out within the framework of the EUROfusion Consortium and the French Research Federation for Fusion Studies and has received funding from the Euratom research and training programme 2014-2018 under grant agreement No 633053. We benefited from HPC resources from GENCI (project 056348), IFERC (project MaCoToP) and from Aix-Marseille Université project Equip@Meso (ANR-10-EQPX-29-01) of the program "Investissements dAvenir" supervised by the Agence Nationale pour la Recherche. This work is part of the project AMICI funded by the Agence Nationale pour la Recherche (ANR-14-CE32-0004-01). 


\section{Appendix: Neoclassical equilibrium}

We recall here for completeness the flux average neoclassical equilibrium equations Hirshman and Sigmar (1981), in SI units:

$$
\begin{aligned}
-e_{s} n_{s}\langle\mathbf{E} \cdot \mathbf{B}\rangle & =-\left\langle\mathbf{B} \cdot \nabla \cdot \boldsymbol{\Pi}_{\mathbf{s}}\right\rangle+\left\langle\mathbf{B} \cdot \mathbf{R}_{\mathbf{s}}\right\rangle \\
0 & =-\left\langle\mathbf{B} \cdot \nabla \cdot \mathbf{\Theta}_{\mathbf{s}}\right\rangle+\left\langle\mathbf{B} \cdot \mathbf{H}_{\mathbf{s}}\right\rangle
\end{aligned}
$$

where $\left\langle\mathbf{B} \cdot \nabla \cdot \boldsymbol{\Pi}_{\mathbf{s}}\right\rangle$ and $\left\langle\mathbf{B} \cdot \nabla \cdot \boldsymbol{\Theta}_{\mathbf{s}}\right\rangle$ are the neoclassical stresses and $\left\langle\mathbf{B} \cdot \mathbf{R}_{\mathbf{s}}\right\rangle$ and $\left\langle\mathbf{B} \cdot \mathbf{H}_{\mathbf{s}}\right\rangle$ the frictions related to the Coulomb collisions. They are expressed as a function of particle flows $\left(u_{1 \| s}=\mathbf{V}_{\mathbf{s}} \cdot \mathbf{B}\right)$ and heat fluxes $\left(u_{2 \|, s}=2 \mathbf{q}_{\mathbf{s}} \cdot \mathbf{B} /\left(5 p_{s}\right)\right)$ as

$$
\begin{aligned}
\left\langle\mathbf{B} \cdot \nabla \cdot \Pi_{\mathbf{s}}\right\rangle & =n_{s} m_{s}\left[\mu_{1}^{s}\left\langle u_{1 \|, s}+S_{1 \theta}^{s}\right\rangle+\mu_{2}^{s}\left\langle u_{2 \|, s}+S_{2 \theta}^{s}\right\rangle\right] \\
\left\langle\mathbf{B} \cdot \nabla \cdot \boldsymbol{\Theta}_{\mathbf{s}}\right\rangle & =n_{s} m_{s}\left[\mu_{2}^{s}\left\langle u_{1 \|, s}+S_{1 \theta}^{s}\right\rangle+\mu_{3}^{s}\left\langle u_{2 \|, s}+S_{2 \theta}^{s}\right\rangle\right] \\
\left\langle\mathbf{B} \cdot \mathbf{R}_{\mathbf{s}}\right\rangle & =n_{s} m_{s} \sum_{s^{\prime}} \Lambda_{11}^{s s^{\prime}} u_{1 \| s^{\prime}}+\Lambda_{12}^{s s^{\prime}} u_{2 \| s^{\prime}} \\
\left\langle\mathbf{B} \cdot \mathbf{H}_{\mathbf{s}}\right\rangle & =n_{s} m_{s} \sum_{s^{\prime}} \Lambda_{21}^{s s^{\prime}} u_{1 \| s^{\prime}}+\Lambda_{22}^{s s^{\prime}} u_{2 \| s^{\prime}}
\end{aligned}
$$

with

$$
\begin{aligned}
\left\langle S_{1 \theta}^{s^{\prime}}\right\rangle & =\frac{\left\langle B^{2}\right\rangle}{\langle\mathbf{B} \cdot \nabla \theta\rangle}\left\langle\left(\mathbf{V}_{\mathbf{s}}^{*}+\mathbf{V}_{\mathbf{E}}\right) \cdot \nabla \theta\right\rangle \\
\left\langle S_{2 \theta}^{s^{\prime}}\right\rangle & =\frac{\left\langle B^{2}\right\rangle}{\langle\mathbf{B} \cdot \nabla \theta\rangle}\left\langle\mathbf{V}_{\text {Ts }}^{*} \cdot \nabla \theta\right\rangle
\end{aligned}
$$

with $\theta$ the poloidal angle.

By using this system of equation $(41,42)$, the parallel heat flows can be expressed as a function of flow quantities (Eq. 15) with the following coefficients:

$$
\begin{array}{rlr}
C_{\|}^{e e} & =1 / A\left[\Lambda_{21}^{i e} \Lambda_{22}^{e i}-\left(\mu_{2}^{e}-\Lambda_{21}^{e e}\right)\left(\mu_{3}^{i}-\Lambda_{22}^{i i}\right)\right] \\
C_{\|}^{e i} & =1 / A\left[\left(\mu_{3}^{i}-\Lambda_{22}^{i i}\right) \Lambda_{21}^{e i}-\left(\mu_{2}^{i}-\Lambda_{21}^{i i}\right) \Lambda_{22}^{e i}\right] \\
C_{\|}^{i e} & =1 / A\left[\left(\mu_{3}^{e}-\Lambda_{22}^{e e}\right) \Lambda_{21}^{i e}-\left(\mu_{2}^{e}-\Lambda_{21}^{e e}\right) \Lambda_{22}^{i e}\right] \\
C_{\|}^{i i} & =1 / A\left[\Lambda_{21}^{e i} \Lambda_{22}^{i e}-\left(\mu_{2}^{i}-\Lambda_{21}^{i i}\right)\left(\mu_{3}^{e}-\Lambda_{22}^{e e}\right)\right]
\end{array}
$$

where:

$$
A=\operatorname{Det}\left(\begin{array}{cc}
\Lambda_{22}^{e e}-\mu_{3}^{e} & \Lambda_{22}^{e i} \\
\Lambda_{22}^{i e} & \Lambda_{22}^{i i}-\mu_{3}^{i}
\end{array}\right)
$$

\section{References}

Ara, G., Basu, B., Coppi, B., Laval, G., Rosenbluth, M., and Waddell, B. (1978). Magnetic reconnection and $\mathrm{m}=1$ oscillations in current carrying plasmas. Annals of Physics, 112(2):443 476, ISSN: 0003-4916, DOI: 10.1016/S0003-4916(78)80007-4, http://www.sciencedirect.com/ science/article/pii/S0003491678800074.

Arcis, N., Escande, D. F., and Ottaviani, M. (2007). Saturation of a tearing mode in zero-beta full magnetohydrodynamics. Physics of Plasmas, 14(3):032308, 032308, DOI: 10.1063/1.2710799, http: //link.aip.org/link/?PHP/14/032308/1. 
Callen, J. D. (2010). Viscous Forces Due To Collisional Parallel Stresses For Extended MHD Codes (EPAPS to Transport equations in tokamak plasmas). Physics of Plasmas, 17(5):056113, 056113, DOI: 10.1063/1.3335486, http://ftp.aip.org/epaps/phys_plasmas/ E-PHPAEN-17-033091/033091php.pdf.

Callen, J. D., QU, W., Siebert, K., Carreras, B., Shaing, K., and Spong, D. (1986). Neoclassical MHD equations, instabilities and transport in tokamaks. Plasma Physics and Controlled Nuclear Fusion Research, II:157-166.

Carrera, R., Hazeltine, R. D., and Kotschenreuther, M. (1986). Island bootstrap current modification of the nonlinear dynamics of the tearing mode. Physics of Fluids, 29(4):899-902, DOI: 10.1063/1.865682, http://link.aip.org/link/?PFL/29/899/1.

Chang, Z., Callen, J. D., Fredrickson, E. D., Budny, R. V., Hegna, C. C., McGuire, K. M., Zarnstorff, M. C., and group, T. (1995). Observation of nonlinear neoclassical pressure-gradient driven tearing modes in TFTR. Phys. Rev. Lett., 74(23):4663-4666, DOI: 10.1103/PhysRevLett.74.4663.

Comisso, L., Grasso, D., and Waelbroeck, F. L. (2015). Extended theory of the taylor problem in the plasmoid-unstable regime. Physics of Plasmas, 22(4):042109, 042109, DOI: http://dx.doi.org/10.1063/1.4918331, http://scitation.aip.org/content/aip/journal/ pop/22/4/10.1063/1.4918331.

Escande, D. F. and Ottaviani, M. (2004). Simple and rigorous solution for the nonlinear tearing mode. Physics Letters A, 323(3-4):278 - 284, ISSN: 0375-9601, DOI: DOI: 10.1016/j.physleta.2004.02.010, http://www.sciencedirect.com/science/article/ B6TVM-4BP3CCD-C/2/6f3dd2e714670f686593c1b009aab66c.

Février, O., Maget, P., Lütjens, H., LUCIANI, J.-F., Decker, J., Giruzzi, G., Reich, M., Beyer, P., Lazzaro, E., and Nowak, S. (2016). First principles fluid modelling of magnetic island stabilization by ECCD. Plasma Physics and Controlled Fusion, 58(4), DOI: 10.1088/0741-3335/58/4/045015, https://hal . archives-ouvertes.fr/hal-01286131.

Fitzpatrick, R. (1995). Helical temperature perturbations associated with tearing modes in tokamak plasmas. Physics of Plasmas, 2(3):825-838, DOI: 10.1063/1.871434, http://link.aip.org/link/ ?PHP/2/825/1.

Furth, H. P., Killen, J., and Rosenbluth, M. (1963). Finite-resistivity instabilities of a sheet pinch. Physics of Fluids, 6(4):459-484.

Glasser, A. H., Greene, J. M., and Johnson, J. L. (1975). Resistive instabilities in general toroidal plasma configurations. Physics of Fluids, 18(7):875-888, DOI: 10.1063/1.861224, http://link.aip.org/ link/?PFL/18/875/1.

Grimm, R. C. and Johnson, J. L. (1972). Viscosity and thermal conductivity effects on the containment of a toroidally confined resistive plasma with flow. Plasma Physics, 14(6):617, http://stacks.iop. org $/ 0032-1028 / 14 / i=6 / a=006$.

Halpern, F. D., Bateman, G., and Kritz, A. H. (2006). Integrated simulations of saturated neoclassical tearing modes in DIII-D, Joint European Torus, and ITER plasmas. Physics of Plasmas (1994present), 13(6):-, 062510, DOI: http://dx.doi.org/10.1063/1.2205829, http://scitation.aip. org/content/aip/journal/pop/13/6/10.1063/1.2205829.

Hennequin, P., Sabot, R., Honoré, C., Hoang, G. T., Garbet, X., Truc, A., Fenzi, C., and Quéméneur, A. (2004). Scaling laws of density fluctuations at high-k on Tore Supra. Plasma Physics and Controlled Fusion, 46(12B):B121, http://stacks.iop.org/0741-3335/46/i=12B/a=011.

Hirshman, S. P. and Sigmar, D. J. (1981). Neoclassical transport of impurities in tokamak plasmas. Nucl. Fusion, 21(9):1079-1201. 
Houlberg, W. A., Shaing, K. C., Hirshman, S. P., and Zarnstorff, M. C. (1997). Bootstrap current and neoclassical transport in tokamaks of arbitrary collisionality and aspect ratio. Physics of Plasmas, 4(9):3230-3242, DOI: 10.1063/1.872465, http://link.aip.org/link/?PHP/4/3230/1.

Ishii, Y., Azumi, M., and Smolyakov, A. (2007). Nonlinear evolution and deformation of driven magnetic islands in rotating plasmas. Nuclear Fusion, 47(8):1024, http://stacks.iop.org/0029-5515/47/i= $8 / a=037$.

Kessel, C. (1994). Bootstrap current in a tokamak. Nucl. Fusion, 34(9):1221-1238.

Konovalov, S. V., Mikhailovskii, A. B., Ozeki, T., Takizuka, T., Shirokov, M. S., and Hayashi, N. (2005). Role of anomalous transport in onset and evolution of neoclassical tearing modes. Plasma Physics and Controlled Fusion, 47(12B):B223, http://stacks .iop.org/0741-3335/47/i=12B/a=S17.

Lazzari, D. D. and Westerhof, E. (2011). The role of asymmetries in the growth and suppression of neoclassical tearing modes. Plasma Physics and Controlled Fusion, 53(3):035020, http://stacks . iop. org $/ 0741-3335 / 53 / i=3 / a=035020$.

Lazzaro, E., Comisso, L., and Valdettaro, L. (2010). Nonlinear response of a neoclassical four-field magnetic reconnection model to localized current drive. Physics of Plasmas (1994-present), 17(5):052509, 052509, DOI: http://dx.doi.org/10.1063/1.3428676, http://scitation.aip.org/content/aip/ journal/pop/17/5/10.1063/1.3428676.

Lazzaro, E. and Nowak, S. (2009). ECCD control of dynamics of asymmetric magnetic islands in a sheared flow. Plasma Physics and Controlled Fusion, 51(3):035005, http://stacks.iop.org/0741-3335/51/ $i=3 / a=035005$.

Loureiro, N. F., Cowley, S. C., Dorland, W. D., Haines, M. G., and Schekochihin, A. A. (2005). x-point collapse and saturation in the nonlinear tearing mode reconnection. Phys. Rev. Lett., 95:235003, DOI: 10.1103/PhysRevLett.95.235003, http://link.aps.org/doi/10.1103/PhysRevLett.95.235003.

Lütjens, H., Bondeson, A., and Sauter, O. (1996). The CHEASE code for toroidal MHD equilibria. Computer Physics Communications, 97(3):219-260, http://www.sciencedirect.com/science/article/ B6TJ5-3VSGD5T-19/2/e429c1260ed10db93fde0078b7cee3e6.

Lütjens, H. and Luciani, J.-F. (2002). Linear and nonlinear thresholds of neoclassical tearing modes in tokamaks. Physics of Plasmas, 9(12):4837-4840, DOI: 10.1063/1.1521717, http://link.aip.org/ link/?PHP/9/4837/1.

Lütjens, H. and Luciani, J.-F. (2005). Saturation levels of neoclassical tearing modes in International Thermonuclear Experimental Reactor plasmas. Physics of Plasmas, 12(8):080703, 080703, DOI: 10.1063/1.2001667, http://link.aip.org/link/?PHP/12/080703/1.

Lütjens, H. and Luciani, J.-F. (2010). XTOR-2F: A fully implicit Newton-Krylov solver applied to nonlinear 3D extended MHD in tokamaks. Journal of Computational Physics, 229(21):8130 8143, ISSN: 0021-9991, DOI: DOI : 10.1016/j.jcp.2010.07.013, http://www.sciencedirect.com/ science/article/B6WHY-50JPNF3-2/2/2787626de93153effa740cca124bcfe7.

Lütjens, H., Luciani, J.-F., and Garbet, X. (2001). Curvature effects on the dynamics of tearing modes in tokamaks. Physics of Plasmas, 8(10):4267-4270, http://link.aip.org/link/?PHP/8/4267/1.

Maget, P., Février, O., Lütjens, H., Luciani, J.-F., and Garbet, X. (2016). Bifurcation of magnetic island saturation controlled by plasma viscosity. Plasma Physics and Controlled Fusion, 58(5), DOI: 10.1088/0741-3335/58/5/055003, https://hal .archives-ouvertes.fr/hal-01292516.

Maget, P., Lütjens, H., Coelho, R., Alper, B., Brix, M., Buratti, P., Buttery, R., la Luna, E. D., Hawkes, N., Huysmans, G., Jenkins, I., Challis, C., Giroud, C., Litaudon, X., Mailloux, J., Ottaviani, M., and Contributors, J.-E. (2010). Modelling of $(2,1)$ NTM threshold in JET advanced scenarios. Nuclear Fusion, 50(4):045004, http://stacks .iop.org/0029-5515/50/i=4/a=045004. 
Maget, P., Lütjens, H., Luciani, J.-F., Garbet, X., Février, O., and Ségui, J.-L. (2014). Bi-fluid and neoclassical effect on a Double-Tearing mode in Tore Supra. Physics of Plasmas (1994-present), 21(6):062504, 062504, DOI: http://dx.doi.org/10.1063/1.4882259, http://scitation.aip.org/ content/aip/journal/pop/21/6/10.1063/1.4882259.

Maget, P., Mellet, N., Lütjens, H., Meshcheriakov, D., and Garbet, X. (2013). Curvature effect on tearing modes in presence of neoclassical friction. Physics of Plasmas, 20(11):112504, 112504, DOI: http://dx.doi.org/10.1063/1.4829038, http://scitation.aip.org/content/aip/ journal/pop/20/11/10.1063/1.4829038.

Maraschek, M. (2012). Control of neoclassical tearing modes. Nuclear Fusion, 52(7):074007, http: //stacks.iop.org/0029-5515/52/i=7/a=074007.

Mellet, N., Maget, P., Lütjens, H., Meshcheriakov, D., and the Tore Supra Team (2013). Neoclassical viscous stress tensor for non-linear MHD simulations with XTOR-2F. Nuclear Fusion, 53(4):043022, http: //stacks.iop.org/0029-5515/53/i=4/a=043022.

Meshcheriakov, D., Maget, P., Lütjens, H., Beyer, P., and Garbet, X. (2012). Linear stability of the tearing mode with two-fluid and curvature effects in tokamaks. Physics of Plasmas, 19(9):092509, 092509, DOI: 10.1063/1.4754000, http://link.aip.org/link/?PHP/19/092509/1.

Meshcheriakov, D., Maget, P., Lütjens, H., Beyer, P., and Garbet, X. (2014). Nonlinear dynamics of the tearing mode with two-fluid and curvature effects in tokamaks. Physics of Plasmas, 21(1):012516, 012516, DOI: http://dx.doi.org/10.1063/1.4863498, http://scitation.aip.org/content/aip/ journal/pop/21/1/10.1063/1.4863498.

Mikhailovskii, A., Shirokov, M., Konovalov, S., and Tsypin, V. (2005). An analytic approach to developing transport threshold models of neoclassical tearing modes in tokamaks. Plasma Physics Reports, 31(5):347-368, ISSN: 1063-780X, DOI: 10.1134/1.1925786, http://dx.doi.org/10.1134/1. 1925786.

Militello, F. and Porcelli, F. (2004). Simple analysis of the nonlinear saturation of the tearing mode. Physics of Plasmas, 11(5):L13-L16, DOI: 10.1063/1.1677089, http://link.aip.org/link/?PHP/ $11 / \mathrm{L} 13 / 1$

Nicolas, T. (2013). Sawtooth driven particle transport in tokamak plasmas. Theses, Ecole Polytechnique X, https://pastel.archives-ouvertes.fr/pastel-00926428.

Ottaviani, M., Porcelli, F., and D. Grasso, D. (2004). Multiple states of nonlinear drift-tearing islands. Phys. Rev. Lett., 93(7):075001, DOI: 10.1103/PhysRevLett.93.075001.

Popov, A. M., Haye, R. J. L., Liu, Y. Q., Murakami, M., Popova, N. N., and Turnbull, A. D. (2002). Simulation of neoclassical tearing modes (NTMs) in the DIII-D tokamak. I. NTM excitation. Physics of Plasmas, 9(10):4205-4228, DOI: 10.1063/1.1505842, http://link.aip.org/link/?PHP/9/4205/1.

Press, W. H., Teukolsky, S. A., Vetterling, W. T., and Flannery, B. P. (2007). Numerical Recipes 3rd Edition: The Art of Scientific Computing. Cambridge University Press, New York, NY, USA, 3 edition, ISBN: 0521880688, 9780521880688.

Rutherford, P. H. (1973). Nonlinear growth of the tearing mode. Physics of Fluids, 16(11):1903-1908, DOI: 10.1063/1.1694232, http://link.aip.org/link/?PFL/16/1903/1.

Sauter, O., Angioni, C., and Lin-Liu, Y. R. (1999). Neoclassical conductivity and bootstrap current formulas for general axisymmetric equilibria and arbitrary collisionality regime. Physics of Plasmas, 6(7):2834-2839, DOI: 10.1063/1.873240, http://link.aip.org/link/?PHP/6/2834/1.

Sauter, O., Angioni, C., and Lin-Liu, Y. R. (2002). Erratum: "neoclassical conductivity and bootstrap current formulas for general axisymmetric equilibria and arbitrary collisionality regime" [phys. plasmas [6], 2834 (1999)]. Physics of Plasmas, 9(12):5140-5140, DOI: 10.1063/1.1517052, http://link .aip. org/link/?PHP/9/5140/1. 
Sauter, O., La Haye, R. J., Chang, Z., Gates, D. A., Kamada, Y., Zohm, H., Bondeson, A., Boucher, D., Callen, J. D., Chu, M. S., Gianakon, T. A., Gruber, O., Harvey, R. W., Hegna, C. C., Lao, L. L., Monticello, D. A., Perkins, F., Pletzer, A., Reiman, A. H., Rosenbluth, M., Strait, E. J., Taylor, T. S., Turnbull, A. D., Waelbroeck, F., Wesley, J. C., Wilson, H. R., and Yoshino, R. (1997). Beta limits in long-pulse tokamak discharges. Physics of Plasmas, 4(5):1654-1664, 1654-1664, DOI: http://dx.doi.org/10.1063/1.872270, http://scitation.aip.org/content/aip/journal/ pop/4/5/10.1063/1.872270.

Smolyakov, A. I., Hirose, A., Lazzaro, E., Re, G. B., and Callen, J. D. (1995). Rotating nonlinear magnetic islands in a tokamak plasma. Physics of Plasmas, 2(5):1581-1598, DOI: 10.1063/1.871308, http://link.aip.org/link/?PHP/2/1581/1.

Waelbroeck, F. L. (1993). Onset of the sawtooth crash. Phys. Rev. Lett., 70:3259-3262, DOI: 10.1103/PhysRevLett.70.3259, http://link.aps.org/doi/10.1103/PhysRevLett.70.3259.

Waelbroeck, F. L. (2007). Nonlinear tearing mode in inhomogeneous plasma: I. unmagnetized islands. Plasma Physics and Controlled Fusion, 49(6):905, http://stacks.iop.org/0741-3335/49/i=6/a= 014.

Wesson, J. (1997). Tokamaks. Oxford Science Publications.

Wilson, H. R., Alexander, M., Connor, J. W., Edwards, A. M., Gates, D., Grüber, O., Hastie, R. J., Hegna, C. C., Hender, T. C., Haye, R. J. L., Lao, L. L., Morris, A. W., Roach, C. M., Strait, E. J., Taylor, T. S., Valovic, M., and Zohm, H. (1996). The collisionality dependence of tokamak $\beta$-limits. Plasma Physics and Controlled Fusion, 38(12A):A149-A163, http://stacks.iop.org/0741-3335/ 38/A149.

Yu, Q. (2010). Linear and nonlinear stability of drift-tearing mode. Nuclear Fusion, 50(2):025014, http: //stacks.iop.org/0029-5515/50/i=2/a=025014.

Yu, Q. and Günter, S. (1998). Modeling of the nonlinear growth of neoclassical tearing modes. Physics of Plasmas (1994-present), 5(11):3924-3928, DOI: http://dx.doi.org/10.1063/1.873112, http:// scitation.aip.org/content/aip/journal/pop/5/11/10.1063/1.873112.

Yu, Q., Günter, S., and Scott, B. D. (2003). Numerical modeling of linear drift-tearing mode stability. Physics of Plasmas, 10(3):797-810, 797-810, DOI: http://dx.doi.org/10.1063/1.1554739, http: //scitation.aip.org/content/aip/journal/pop/10/3/10.1063/1.1554739. 